\title{
Transcriptome analysis of thermophilic methylotrophic Bacillus methanolicus MGA3 using RNA-sequencing provides detailed insights into its previously uncharted transcriptional landscape
}

Marta Irla ${ }^{1 \dagger}$, Armin Neshat $^{2 \dagger}$, Trygve Brautaset ${ }^{3,4}$, Christian Rückert $^{2,5}$, Jörn Kalinowski ${ }^{2,5}$ and Volker F Wendisch ${ }^{1 *}$

\begin{abstract}
Background: Bacillus methanolicus MGA3 is a thermophilic, facultative ribulose monophosphate (RuMP) cycle methylotroph. Together with its ability to produce high yields of amino acids, the relevance of this microorganism as a promising candidate for biotechnological applications is evident. The B. methanolicus MGA3 genome consists of a 3,337,035 nucleotides (nt) circular chromosome, the 19,174 nt plasmid pBM19 and the 68,999 nt plasmid pBM69. 3,218 protein-coding regions were annotated on the chromosome, 22 on pBM19 and 82 on pBM69. In the present study, the RNA-seq approach was used to comprehensively investigate the transcriptome of $B$. methanolicus MGA3 in order to improve the genome annotation, identify novel transcripts, analyze conserved sequence motifs involved in gene expression and reveal operon structures. For this aim, two different cDNA library preparation methods were applied: one which allows characterization of the whole transcriptome and another which includes enrichment of primary transcript 5'-ends.

Results: Analysis of the primary transcriptome data enabled the detection of 2,167 putative transcription start sites (TSSs) which were categorized into 1,642 TSSs located in the upstream region (5'-UTR) of known protein-coding genes and 525 TSSs of novel antisense, intragenic, or intergenic transcripts. Firstly, 14 wrongly annotated translation start sites (TLSs) were corrected based on primary transcriptome data. Further investigation of the identified 5'-UTRs resulted in the detailed characterization of their length distribution and the detection of 75 hitherto unknown cis-regulatory RNA elements. Moreover, the exact TSSs positions were utilized to define conserved sequence motifs for translation start sites, ribosome binding sites and promoters in B. methanolicus MGA3. Based on the whole transcriptome data set, novel transcripts, operon structures and mRNA abundances were determined. The analysis of the operon structures revealed that almost half of the genes are transcribed monocistronically (940), whereas 1,164 genes are organized in 381 operons. Several of the genes related to methylotrophy had highly abundant transcripts.
\end{abstract}

Conclusion: The extensive insights into the transcriptional landscape of B. methanolicus MGA3, gained in this study, represent a valuable foundation for further comparative quantitative transcriptome analyses and possibly also for the development of molecular biology tools which at present are very limited for this organism.

Keywords: Bacillus methanolicus, Methylotrophy, RNA-sequencing, Transcriptome analysis, Conserved sequence motifs, Operon structures, Regulatory RNA, Transcript abundances, Transcriptional start sites, Ribosome binding sites

\footnotetext{
*Correspondence: volker.wendisch@uni-bielefeld.de

${ }^{\dagger}$ Equal contributors

${ }^{1}$ Genetics of Prokaryotes, Faculty of Biology \& Center for Biotechnology,

Bielefeld University, Universitätsstr. 25, 33615 Bielefeld, Germany

Full list of author information is available at the end of the article
} 


\section{Background}

Bacillus methanolicus MGA3, isolated from freshwater marsh soil, is an aerobic, Gram-positive, endosporeforming, rod-shaped bacterium auxotrophic for biotin and vitamin $B_{12}[1,2]$. This thermophilic organism grows in a temperature range between 37 and $60^{\circ} \mathrm{C}$ with an optimum between $50-53^{\circ} \mathrm{C}[1,2]$. B. methanolicus MGA3 is able to utilize methanol as its sole carbon and energy source via the RuMP assimilation cycle. Moreover, B. methanolicus was reported to secrete 55 grams of glutamate per liter in fed-batch cultures with methanol as sole carbon and ammonium as nitrogen source $[3,4]$. These characteristics indicate that B. methanolicus is a valuable candidate for amino acid production from methanol which in turn is an interesting alternative to the conventional feedstocks used in the biotechnological industry, i.e. mainly molasses and starch hydrolysates. Methanol is advantageous since it is abundant, its price does neither depend on the climate nor on agricultural politics, it is a pure compound and it reduces the risk of contamination in fermentations due to its toxicity towards numerous microbial species [5,6]. Fermentations with the comparatively reduced methanol are characterized by high oxygen demands and heating of bioreactors causing high cooling costs. However, this is less relevant for the thermophilic B. methanolicus $[5,6]$.

Most molecular biology work on B. methanolicus has been devoted to enzymes relevant to methylotrophy. B. methanolicus possesses three active variants of NADdependent methanol dehydrogenase, two chromosomeencoded and one pBM19-encoded, for oxidation of methanol to formaldehyde $[7,8]$, and one activator protein ACT [9]. Formaldehyde is assimilated in the RuMP cycle. The enzymes for condensation of formaldehyde with ribulose to fructose 6-phosphate, 3-hexulose 6-phosphate synthase and 6-phospho-hexuloisomerase, are only encoded on the chromosome [10]. The majority of genes encoding the enzymes of the RuMP cycle in B. methanolicus MGA3 is present in the genome in two copies, chromosome-encoded and pBM19-encoded [10,11]. Although all of the genes coding for the transketolase variant of the regeneration phase have their chromosomal version, pBM19-cured B. methanolicus MGA3 strain MGA3-A6 shows a methylotrophy deficient phenotype [10]. The five RuMP cycle genes and $m d h$ encoded on pBM19 are induced during growth on methanol. The variants of the enzymes do not always differ in their biochemical characteristics, e.g. the transketolases [12]. By contrast, the fructose 1,6-bisphosphatases differ since the major fructose 1,6-bisphosphatase $\mathrm{GlpX}^{\mathrm{C}}$ is encoded on the chromosome, while pBM19-encoded $\mathrm{GlpX}^{\mathrm{P}}$ is also active as sedoheptulose 1,7-bisphosphatase in the eponymous regeneration variant of the RuMP cycle [13]. Similarly, whereas both fructose 1,6- bisphosphate aldolases $\mathrm{FBA}^{\mathrm{C}}$ and $\mathrm{FBA}^{\mathrm{P}}$ are also active as sedoheptulose 1,7-bisphosphate aldolases, their kinetic parameters allowed to distinguish $\mathrm{FBA}^{\mathrm{C}}$ as major glycolytic $\mathrm{FBA}$ and $\mathrm{FBA}^{\mathrm{P}}$ as major gluconeogenic FBA [14].

Sequencing of the B. methanolicus MGA3 genome, comparative DNA microarray transcriptome analyses and proteome studies have increased our understanding of the genetic and regulatory background for methanol metabolism in B. methanolicus MGA3 [10,11,15,16]. However, to date a comprehensive transcriptome analysis of $B$. methanolicus MGA3 is still missing. Therefore, transcriptome sequencing (RNA-seq) [17,18] was here used for the complete analysis of the mostly uncharted transcriptional landscape of $B$. methanolicus MGA3. Cultivations of $B$. methanolicus MGA3 were performed in several different conditions in order to capture a wide range of transcripts. RNA-seq data for the analysis of the primary transcriptome and the whole transcriptome were generated to gain insights about the exact positions of transcription start sites, length distribution of $5^{\prime}$-UTRs, consensus promoter and ribosome binding site (RBS) motifs, transcriptional organization of operons, and mRNA abundances. Moreover, cis-regulatory RNA elements and novel transcripts in intergenic regions, intragenic sense transcripts of annotated genes as well as antisense transcripts located within known genes were identified.

\section{Methods}

\section{Cultivation of B. methanolicus MGA3 in different growth media}

B. methanolicus wild-type strain MGA3 was used as the model organism in all growth experiments. The fermenter minimal medium was a derivative of MVcMY medium but instead of vitamin solution, $6 \mathrm{mg} / \mathrm{liter}$ d-biotin and $0.01 \mathrm{mg} /$ liter vitamin $\mathrm{B}_{12}$ were used [19]. If not stated differently $200 \mathrm{mM}$ methanol was used as carbon source both for flask and fermenter cultivations. The overnight cultures grown in $500 \mathrm{ml}$ shake flasks with $50 \mathrm{ml}$ of working volume of MVcMY minimal medium at $50^{\circ} \mathrm{C}$ and $200 \mathrm{rpm}$ as described by Brautaset and coworkers [4] were used as inoculum for fermentations at a start optical density $\left(\mathrm{OD}_{600}\right)$ of 0.15 . The fermentations were performed in $1 \mathrm{~L}$ Biostat Q bioreactors with working volume of the minimal medium of $800 \mathrm{ml}$ (Sartorius, Göttingen, Germany). The standard cultivation conditions were: temperature at $50^{\circ} \mathrm{C}$, the dissolved oxygen level at $30 \%$ and the $\mathrm{pH}$ maintained by automatic addition of $12.5 \%$ $\mathrm{NH}_{4} \mathrm{OH}$ (wt/vol) at 6.5. An antifoam agent (Antifoam 204, Sigma-Aldrich, St. Louis, MO, USA) was added at an initial concentration of $0.1 \%$ (vol/vol). Fermentations were performed with initial agitation at level 
of $120 \mathrm{rpm}$ and with no initial aeration. The level of dissolved oxygen was controlled by automatic adjustment of the aeration rate up to $0.75 \mathrm{NL} \cdot \mathrm{min}^{-1}$ and followed by an increase of the stirrer speed up to $1200 \mathrm{rpm}$.

In order to induce the transcription of as many genes as possible, B. methanolicus was cultivated in 16 different conditions; 13 cultivations in bioreactors and 3 cultivations in shake flasks. For induction of $\mathrm{pH}$ shock response the bacterium was cultivated in a $\mathrm{pH}$ range between 5.5 and 7.5 with 0.5 intervals, for oxygen stress conditions the dissolved oxygen was maintained at levels $5 \%, 20 \%$ and $50 \%$, and osmotic stress was induced by addition of $300 \mathrm{mM}$ sorbitol or $160 \mathrm{mM} \mathrm{NaCl}$. Additionally, $50 \mathrm{mM}$ mannitol or $50 \mathrm{mM}$ glucose were used as carbon sources and $16 \mathrm{mM}$ glutamine was used as sole nitrogen source. For the latter fermentation, $1 \mathrm{~N}$ $\mathrm{NaOH}$ was used to maintain $\mathrm{pH}$ at a level of 6.5. Apart from above described changes, the fermentations were performed in standard conditions. Additionally, three flask cultivations were performed in minimal medium MVcMY with $200 \mathrm{mM}$ methanol, $50 \mathrm{mM}$ glucose or $50 \mathrm{mM}$ mannitol as the sole carbon source. In each case, $20 \mathrm{~mL}$ of sample was collected in the exponential phase to $50 \mathrm{~mL}$ falcons filled with ice and centrifuged at $4^{\circ} \mathrm{C}$ for $10 \mathrm{~min}$ at $3.220 \mathrm{x}$ g. For the standard cultivation conditions the samples were additionally collected at the beginning and at the end of the exponential phase. The resulting cell pellets were frozen in liquid nitrogen and stored at $-80^{\circ} \mathrm{C}$ until further use.

\section{Total RNA isolation}

For the extraction of total RNA the NucleoSpin RNA isolation kit (MACHEREY-NAGEL, Düren, Germany) and the RNase-free DNase set (QIAGEN, Hilden, Germany) were used according to the manufacturer's instructions. The total RNA was isolated individually for each cultivation condition. The quality and quantity of the isolated RNA was assessed by PCR with genespecific primers (Additional file 1: Table S1) and with capillary gel electrophoresis (Agilent Bioanalyzer 2100 system using the Agilent RNA 6000 Pico kit; Agilent Technologies, Böblingen, Germany). The extracted RNA samples were pooled in equal parts and the pool of total RNA was subsequently used for the preparation of two different cDNA libraries.

\section{Preparation of two different cDNA libraries for high- throughput sequencing}

The cDNA libraries of $B$. methanolicus MGA3 were prepared according to two different protocols. One approach focused on the enrichment of 5 '-ends of primary transcripts, while the other method allowed the analysis of the whole transcriptome. Both cDNA library preparation protocols have been previously described in detail [17]. In the present study, the experimental workflow was changed at two steps. The first difference is that the amplification of cDNA fragments was customized by adding the PCR additive betaine to a final concentration of $2.7 \mathrm{M}$ in each PCR reaction. Secondly, after cDNA amplification the two libraries were purified and size-selected via gel electrophoresis for fragment sizes between 100 and $800 \mathrm{bp}$. The cutoff of $100 \mathrm{bp}$ was chosen to reduce adapter dimers in the finished library. Due to the fact that the preparation workflow involves the use of two adapters, which together have a length of $66 \mathrm{nt}$, only transcripts smaller than $40 \mathrm{nt}$ are not present in the final RNA seq data. Subsequently, each cDNA library was sequenced according to the manufacturer's instruction on a single flow cell using TruSeq kits (Illumina, San Diego, CA, USA) on a MiSeq Desktop Sequencer system (Illumina, San Diego, CA, USA). Sequencing was performed in single-read mode with 50 nt read length for the enriched 5 '-ends cDNA library and in paired-end mode with 25 nt read length for the whole transcriptome cDNA library. The base calling was realized with the Illumina instrument software.

\section{Mapping of the generated RNA-seq data to reference sequences}

In order to perform further analyses on the RNA-seq data, the generated reads were mapped to the $B$. methanolicus MGA3 reference sequences of the chromosome and the two plasmids pBM69 and pBM19 (GenBank accession no. CP007739, CP007740 and CP007741). For this, two different strategies were utilized for the enriched 5 '-ends of primary transcripts RNA-seq data and the whole transcriptome data set. In a preprocessing step, the last bases of all sequence reads from the enriched 5 '-ends approach were trimmed to a final length of $20 \mathrm{nt}$. This was mainly done because only the read starts of this data set are important for subsequent analyses, but also because of the higher error rate which is often displayed by the last bases of sequence reads [20]. Next, these trimmed reads were mapped to the B. methanolicus MGA3 reference sequences using the exact mapping algorithm SARUMAN [21] and allowing a single mismatch to occur in the alignment of each read. In case of the reads belonging to the whole transcriptome RNA-seq data set, the forward and reverse read were combined to one read, containing the reference sequence as an insert, if both reads were present and in inverse orientation in a maximal distance of $1 \mathrm{~kb}$ to each other. Sequence reads not fulfilling this criterion were retained as single mapping reads. Paired mappings with a distance larger than $1 \mathrm{~kb}$ were discarded from subsequent analyses. For the visualization of the mapped short reads, the Java software ReadXplorer [22] was used. 


\section{Determination of putative transcription start sites using 5'-ends of enriched primary transcripts}

The identification of putative transcription start sites was performed based on the method described previously [17], but with a recently developed transcriptome analysis module which will be integrated into the software ReadXplorer in the near future [www.readxplorer. org, 22]. To determine putative TSSs, the RNA-seq data of enriched native $5^{\prime}$-ends was used. First, for each strand and position of the B. methanolicus MGA3 reference sequences, all reads starts at each genomic position were counted. Putative TSSs were automatically and systematically called if the read starts at the analyzed genomic position fulfilled three criteria. A putative TSS was identified if the number of read starts at the genomic position exceeded the background threshold $T$ and if the ratio of read starts at this position to read starts at the previous position was higher than the ratio $R$. Additionally, the putative TSS was only automatically identified if it was located within a maximal distance $X$ between the putative TSS and the next TLS. For every identified candidate TSS, additional information on the genomic vicinity regarding next upstream, downstream, and overlapping features on both strands were taken into account. In particular, the gene identifier, gene type (protein-coding gene, rRNA, tRNA), TSS distance to feature start, and TSS distance to feature stop were reported. Additionally, the putative TSSs set, which was obtained by automated analysis, was manually cross-checked with the complete enriched 5 '-ends dataset. This revision allowed to disregard falsepositive TSSs and to add putative true-positives which are below the automatic detection limit. A putative TSS was assumed to be false-positive and disregarded if no clear accumulation of read starts was observed at the particular genomic position and additionally the putative TSS was detected within an uneven gradient of accumulated read starts. The described combination often applied to putative TSSs detected within a coding region and/or with a relatively high amount of accumulated reads $(>100)$, where the parameters used for automated TSSs detection are not effective. A putative TSS was manually added if only a single read difference to one of the thresholds $T$ or $R$ was observed and if additionally the whole transcriptome RNA-seq data confirmed the assumption of a valid transcript at the examined genomic position. In addition, most novel intergenic features were manually added as these understandably did not match the chosen maximal distance cutoff between TSSs and next TLSs. Following the manual verification, the determined set of putative TSSs was divided into subsets depending on their genomic context within the annotated genome of B. methanolicus MGA3 (Figure 1).

\section{Identification of operon structures using whole transcriptome cDNA library RNA-seq data}

To determine operon structures of $B$. methanolicus MGA3, the generated whole transcriptome cDNA library RNA-seq data was used. In the present study, an approach was applied which is based on a previously described method [17] and which soon will be integrated into the software ReadXplorer [www.readxplorer.org, 22 ]. For the identification of operons, it was examined if a gene and the next downstream gene in sense direction are connected by a number of paired mappings exceeding the background threshold $T$ for this data set. The background threshold $T$ was set to 4 after manual analysis of different values. Thus, two genes oriented in sense direction were combined to an operon unit if at least 4 paired mappings were bridging over the intergenic region of those genes. This process was progressively performed for all genes located on the chromosome and the two plasmids of $B$. methanolicus MGA3. All consecutive gene pairs were combined, whereas the first gene of the operon was always considered to constitute the primary operon. In most cases a TSS could be determined for the first gene of these primary operons. Suboperons were called if TSSs were detected for genes within the primary operon and defined as subset of genes preceded by the TSS present within the primary operon. Additionally, the automatically generated operon set was manually cross-checked with the complete whole transcriptome RNA-seq data. In this context, genes were manually added to an operon if only a single read difference to the thresholds $T$ was observed and if additionally the genes were functionally related.

\section{Detection of conserved sequence motifs in Bacillus methanolicus MGA3}

To identify conserved sequence motifs for translation starts, ribosome binding sites and promoter regions of B. methanolicus MGA3, the motif-finding program Improbizer [23] and the visualization tool WebLogo [24] were used with default settings. The input differed according to the respective analysis context: For the determination of TLSs and promoter motifs all genes with an identified $5^{\prime}$-UTR were considered, while for the RBSs motifs only $5^{\prime}$-UTRs longer than 9 nucleotides were analyzed. The identified motifs are represented in the text by upper or lower case bases dependent on their conservation. An upper case letter is used if the nucleotide occurs in more than $80 \%$ of all analyzed sequences, whereas a lower case letter is used, if the base appears in more than $40 \%$, but less than $80 \%$ of all cases. If a base occurs less than $40 \%$ at a certain position, a lower case $n$ is used. 


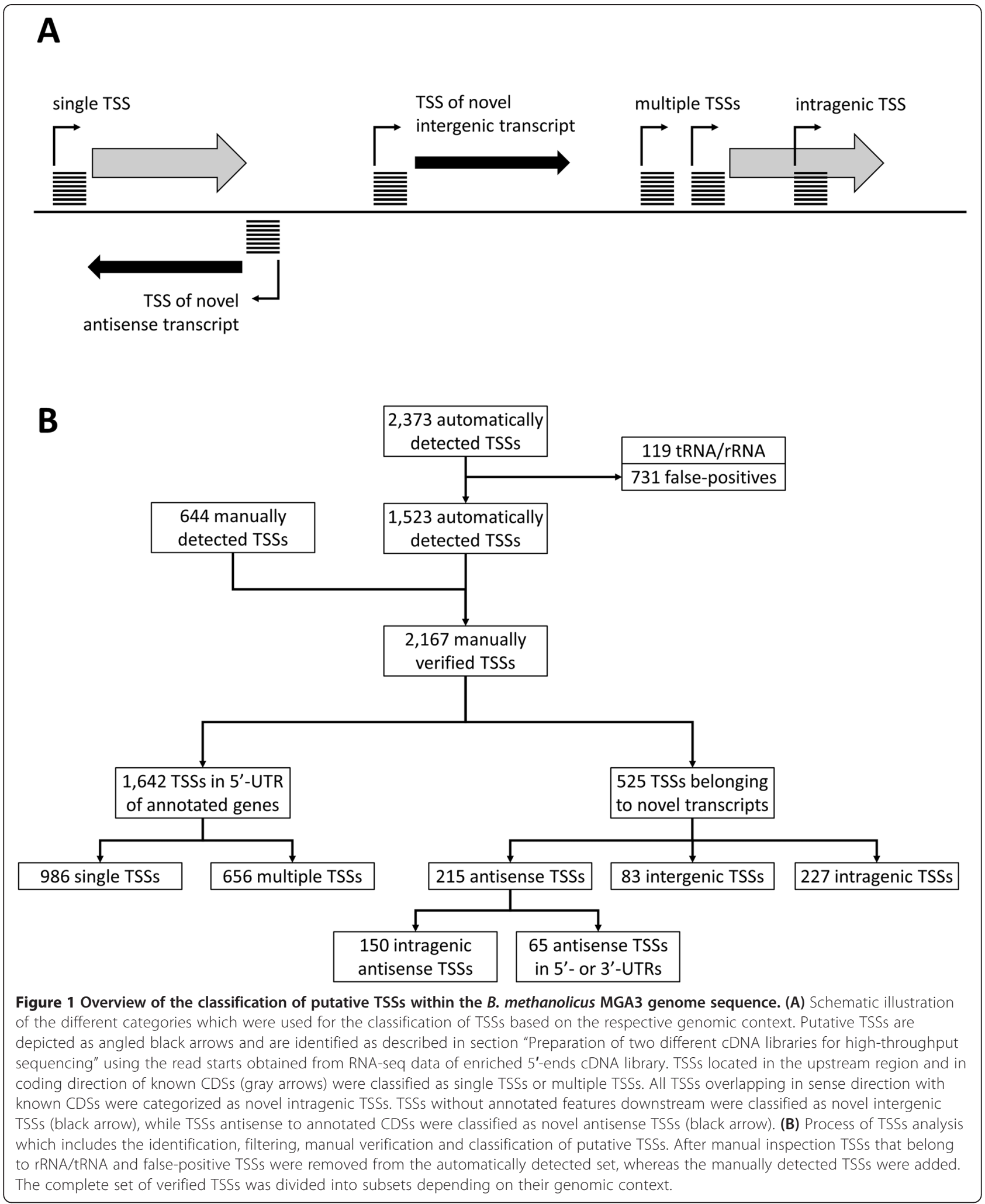




\section{Calculation of transcript abundances in Bacillus methanolicus MGA3}

To determine and compare transcript abundances across different features in B. methanolicus MGA3, the generated whole transcriptome data had to be normalized. One of the most basic methods for the normalization of RNA-seq data is referred to as reads per kilobase of coding DNA sequence (CDS) per million mapped reads (RPKM) [25]. To account for fragmentation and PCR biases during the cDNA library preparation, log-RPKM values were determined and used for comparison of transcript abundances in the present study. For this the following calculations were performed:

$$
\bar{x}=\frac{\sum_{p_{i=1}}^{N} \ln \left(r s_{p_{i}}\right)}{N}
$$

For each single nucleotide position $p_{i}$ belonging to a transcript, the natural logarithm of the number of mapped read starts at that position $\left(r s_{p_{i}}\right)$ was calculated and added up, if at least one read was mapped. Next, the arithmetic mean $\bar{x}$ of these data was calculated and this number was used in the following formula:

$$
R_{\text {norm }}=e^{\bar{x}} N
$$

to finally obtain a normalized quantity of reads per CDS $\left(R_{\text {norm }}\right)$. This number of normalized reads per CDS was than utilized in the common formula for RPKM determination.

\section{Results}

Cultivations of $B$. methanolicus MGA3 under different conditions for analysis of the primary and whole transcriptome

The comprehensive characterization of 'static' bacterial transcriptomes requires the expression of as many genes as possible in order to obtain a pool of different transcripts. In this study, B. methanolicus MGA3 was subjected to 13 bioreactor fermentations and three flask cultivations with different carbon sources (200 mM methanol, $50 \mathrm{mM}$ mannitol and $50 \mathrm{mM}$ glucose) and under various growth conditions. The standard 1-L bioreactor cultivation was carried out at $\mathrm{pH} 6.5, \mathrm{pO}_{2} 30 \%$, temperature $50^{\circ} \mathrm{C}$ and with $200 \mathrm{mM}$ methanol as carbon source and a working volume of $800 \mathrm{~mL}$. The changes in these standard settings included $\mathrm{pH}$ varying between 5.5 and 7.5, $\mathrm{pO}_{2}$ at levels of $5 \%, 20 \%$ and $50 \%$, use of $50 \mathrm{mM}$ glucose or mannitol instead of methanol as sole carbon source and $16 \mathrm{mM}$ glutamine as sole nitrogen source, and application of osmotic stress by addition of $300 \mathrm{mM}$ sorbitol or $160 \mathrm{mM} \mathrm{NaCl}$. The samples were taken at three time points $(3,4$ and 8 hours with the respective $\mathrm{OD}_{600}$ of 1.6, 2.4 and 12.1) during bioreactor cultivation in standard conditions and only at one time point in the exponential phase for the other cultivations $\left(\mathrm{OD}_{600}\right.$ of 2.5 to 6$)$. The growth pattern under the standard conditions (high growth rate of $0.52 \mathrm{~h}^{-1}$, decline of biomass concentration after reaching maximum $\mathrm{OD}_{600}$ of 13.5 corresponding to $2.97 \mathrm{~g} \mathrm{CDW/L)} \mathrm{was} \mathrm{compar-}$ able to the one previously described for B. methanolicus [26] (Additional file 2: Table S2). During cultivation on glucose as the sole carbon and energy source, sporulation was observed which is in accordance with previous findings [26]. As expected, growth rates were lower under stress conditions ( $\mathrm{pH}$, osmotic and oxygen stress) than for standard cultivation conditions. Taken together, these results indicate that $B$. methanolicus MGA3 was submitted to various different growth conditions, thus, quite different gene expression patterns involving most of the genes on the B. methanolicus genome may be expected.

\section{Sequencing of CDNA libraries and mapping to the B. methanolicus MGA3 reference sequences}

After RNA isolation and pooling, two cDNA libraries (whole transcriptome and enriched for $5^{\prime}$-ends of primary transcripts) were prepared as described previously [17]. The cDNA libraries were sequenced on a single flow cell on a MiSeq Desktop Sequencer system. About 3.28 million sequence reads were obtained from enriched 5 '-ends of primary transcripts and about 4.24 million sequence reads for the whole transcriptome cDNA library (Table 1). Subsequently, the obtained reads were mapped to the chromosome and the two plasmids of B. methanolicus MGA3 using the exact alignment algorithm SARUMAN [21]. About 1.30 million of 3.28 million sequence reads for the enriched 5 '-ends of primary transcripts library and about 4.20 out of the 4.24 million sequence reads of the whole transcriptome library were mapped to the reference sequences. Of those mapped reads, 1.25 million reads from enriched 5 '-ends of primary transcripts and 2.60 million reads from the whole transcriptome mapped uniquely. Reads of the whole transcriptome data were combined to one read with the reference sequence as insert, if the forward and reverse read were present and maximal distance to each other was $1 \mathrm{~kb}$ (Table 1$)$.

\section{Detection of putative transcription start sites from RNA- seq data of enriched 5'-ends of primary transcripts}

Putative TSSs were derived from the mapped read starts of enriched 5 '-ends of primary transcripts. Based on background threshold $T$ (empirically set to 6), ratio $R$ (6) and distance cutoff $X$ of 500 bp that showed a good signal-to-noise ratio and specificity after manual inspection of randomly selected TSSs from the result set, 2,373 TSSs were detected (Figure 1). After a complete review of the TSSs set and comparison to the raw RNA-seq 
Table 1 Sequencing and mapping results for the CDNA libraries of $B$. methanolicus MGA3

\begin{tabular}{|c|c|c|c|}
\hline & Reference & 5'-ends of primary transcripts & Whole transcriptome \\
\hline Sequence reads & & $3,278,605$ & $4,241,887$ \\
\hline \multirow[t]{4}{*}{ Mapping reads } & Chromosome & $1,189,365$ & $3,857,244$ \\
\hline & pBM19 & 91,185 & 315,572 \\
\hline & pBM69 & 17,709 & 28,341 \\
\hline & Total & $1,298,259$ & $4,201,157$ \\
\hline \multirow[t]{4}{*}{ Unique matches (single reads) } & Chromosome & $1,141,761$ & 942,678 \\
\hline & pBM19 & 91,169 & 68,844 \\
\hline & pBM69 & 17,686 & 7,793 \\
\hline & Total & $1,250,616$ & $1,019,315$ \\
\hline \multirow[t]{4}{*}{ Unique matches (combined reads) } & Chromosome & - & $1,444,584$ \\
\hline & pBM19 & - & 123,354 \\
\hline & pBM69 & - & 10,172 \\
\hline & Total & - & $1,578,110$ \\
\hline
\end{tabular}

data set, 644 manually determined TSSs were added to the group of TSSs, while 731 false-positive TSSs and 119 TSSs located upstream of tRNA or rRNA genes were removed. These 2,167 manually verified TSSs were assigned to subgroups of TSSs that are located in the $5^{\prime}$ UTR of annotated genes $(1,642)$ and TSSs that belong to novel transcripts (525). The 1,642 TSSs found in the $5^{\prime}$ UTRs of annotated genes were further categorized into transcripts for which only one or several TSSs could be determined, thus resulting in a set of 986 single TSSs and a set of 656 multiple TSSs, originating from 283 genes (Figure 1). Of the 525 TSSs belonging to novel transcripts, 215 were located in antisense orientation to an annotated gene or its untranslated region, 83 TSSs were intergenic and 227 were intragenic TSSs. The intragenic TSS within the first half of CDSs were used to correct 14 CDSs starts, which were previously wrongly annotated (Additional file 3: Table S3).

\section{Determination and length distribution of 5 -untranslated regions in $B$. methanolicus MGA3}

The distances of 1,642 TSSs belonging to the leader regions of protein-coding genes to the next TLSs revealed a median $5^{\prime}$-UTR length of 51 nt (Figure 2). More than $99 \%$ of the transcripts analyzed in this study have $5^{\prime}$ UTR leader sequences of $\geq 10$ nt. $22 \%$ of transcripts

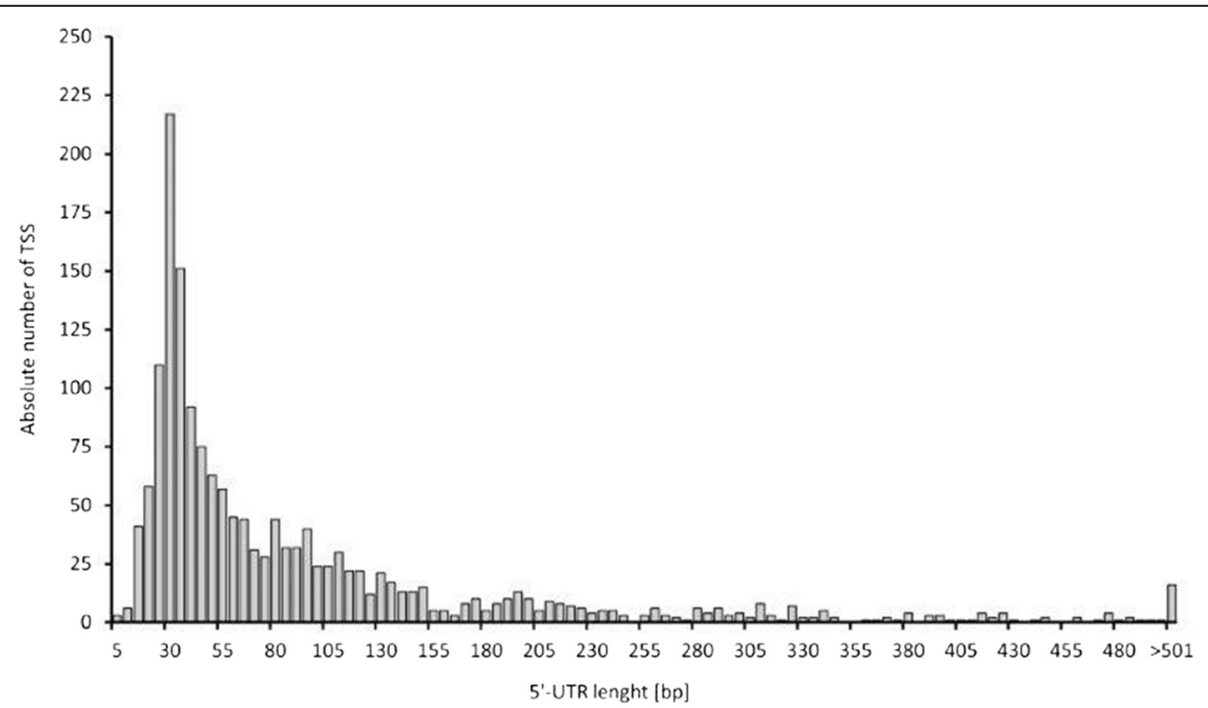

Figure 2 Absolute number of identified transcription start sites in correlation to the length of their $5^{\prime}$-untranslated regions (5'-UTRs). The 1,642 TSSs located upstream or in coding direction of known CDSs were used to determine the length of the $5^{\prime}-U T R s$ for each CDS. The $5^{\prime}$-UTR length was calculated as the distance between an identified TSS to the next TLS. The absolute number of TSSs is grouped in 5 bp intervals of 5'-UTR lengths (1-5, 6-10, 11-15 etc.), whereas the most distant right bar represents all 5'-UTRs longer than 500 bases. 
showed a 5'-UTR length of $26-35$ nt and $25 \%$ of transcripts contain leader sequences longer than $100 \mathrm{nt}$, which suggests that they might contain regulatory RNA structures [27]. Only six transcripts $(<0.5 \%)$ possess a 5 '-UTR shorter than $10 \mathrm{nt}$, which implies that they do not retain a (complete) RBS. However, no leaderless transcript was found for B. methanolicus MGA3 since all transcripts had 5'-UTRs $\geq 2$ nt. Only two transcripts showed leaders shorter than $5 \mathrm{nt}$. The corresponding genes are BMMGA3_05465 encoding the CtaG protein which participates in the formation of active cytochrome caa3 and sigF encoding the sporulation-specific sigma factor $\sigma^{\mathrm{F}}[28]$.

\section{Identification of ribosome binding sites in B. methanolicus MGA3}

For the determination of the nucleotide distribution in TLSs of B. methanolicus MGA3, the initiation codons of 1,270 genes, for which a $5^{\prime}$-UTR was identified (section "Detection of putative transcription start sites from RNA-seq data of enriched 5' ends of primary transcripts"), were analyzed. For genes with more than one TSS, the upstream sequence of the respective gene was only included once in the analysis. From this set, 1,264 upstream regions of genes with a $5^{\prime}$-UTR longer than 9 nt were used to identify a conserved ribosome binding site sequence using the tools Improbizer [23] and WebLogo [24]. The determined RBS motif of B. methanolicus MGA3 (Figure 3) was present in 1,201 of 1,264 upstream regions (95.0\%). The preferred translational start codon was ATG (959 genes, 75.5\%), followed by TTG (177 genes, 13.9\%) and GTG (134 genes, 10.6\%), while CTG was not found in such position. The

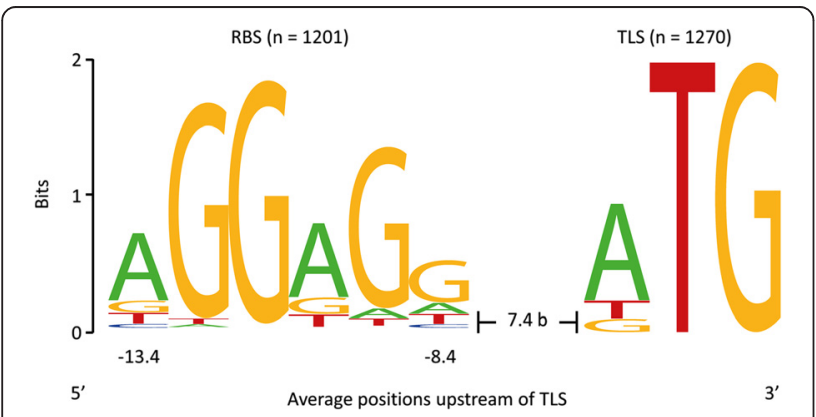

Figure 3 Distribution of nucleotides within the ribosome binding sites and translation starts of $B$. methanolicus MGA3. The analysis of the nucleotide distribution in translation start sites and ribosome binding sites of $B$. methanolicus MGA3 was based on the TLS and upstream regions of genes for which a $5^{\prime}$-UTR was identified in the present study. The conserved TLSs and RBSs motifs were determined by using the motif-finding program Improbizer [23]. The conservation of a specific nucleotide at certain position is measured in bits and represented in the illustration by the size of the nucleotide. The depicted sequence logo was created with the software WebLogo [24]. determined RBS motif in B. methanolicus MGA3 is aGGaGg with the three guanines depicted in capital letters being present in approximately $90 \%$ of the analyzed sequences (Figure 3). The distance between the RBSs and the TLSs varies between 5 to 10 nucleotides (97.1\%) with an average spacing of 7.4 bases. The assumed optimal interval of 7 or 8 bases accounts for $58.5 \%$ of the determined spacers in B. methanolicus MGA3.

\section{Identification of promoter motifs in B. methanolicus MGA3}

The upstream regions of the 1,642 TSSs, which were identified at the 5'-UTR of annotated genes (see section "Detection of putative transcription start sites from RNA-seq data of enriched 5 ' ends of primary transcripts") were searched for conserved motifs within 70 bases upstream of each TSS using Improbizer [23]. The -10 hexamer sequence TAtaaT was identified in 1,619 of the upstream sequences (98.6\%) (Figure 4) and in 33\% of the sequences the motif TGN was present. The distance between an identified -10 region to the corresponding TSS ranges from 4 to $10 \mathrm{nt}$ and is in average 6.7 bases in length. Upstream of an identified -10 hexamer sequence, a weakly conserved -35 motif ttgana was found in 1,616 (98.4\%) upstream sequences (Figure 4). The first three bases of the motif are present in approximately $70 \%$ of the sequences and the following three bases in about $46 \%$. The average distances between the -10 and the -35 regions was 16.6 bases in B. methanolicus MGA3.

When the genes with 1,642 detected upstream regions were subdivided into the four COG (Clusters of Orthologous Groups) categories: information storage and processing (1), cellular processes and signaling (2), metabolism (3) and unknown function or poorly characterized (4), nearly the same -10 and -35 motifs as described above were present in each group (data not shown). Slight deviations were detected only for the weaker conserved positions of the two motifs. In group 1 , a weakly conserved guanine was observed at the second position upstream of the -10 region. In groups 1 and 2, thymine at the last position of the -35 region is slightly more conserved than adenine. The first three bases (ttg) of the -35 region were marginally less conserved in group 4.

The 21 transcripts with the highest abundance (section "Transcript abundances of B. methanolicus MGA3") and with an available upstream region showed conserved -10 and -35 elements, however, certain differences to the above described consensus motifs were found: The third position of the -10 hexamer showed no conservation at all (TAnaaT), while the first three bases of the -35 region (tTg) and especially the second position were slightly more conserved (data not shown). 


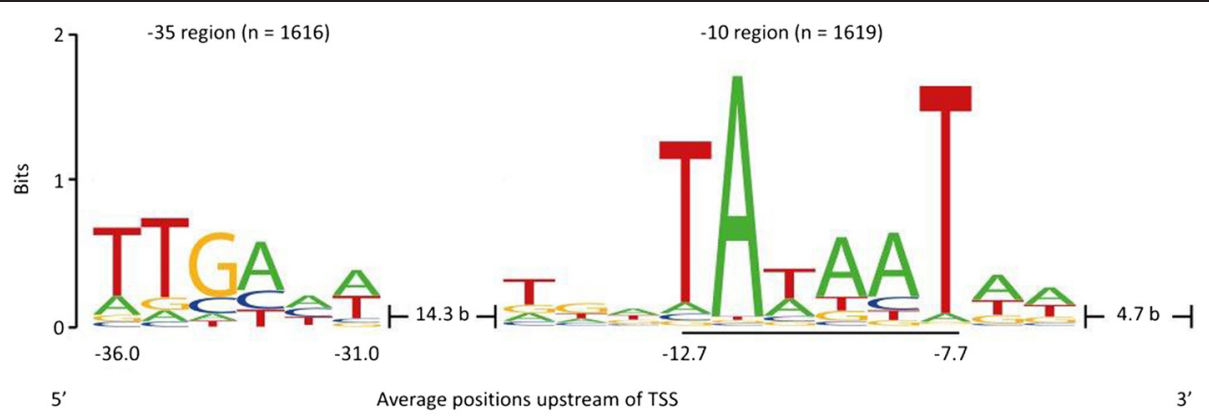

Figure 4 Distribution of nucleotides within the -10 and -35 regions of $B$. methanolicus MGA3 promoter regions. The conserved sequences were determined by using the Improbizer motif-finding program [23]. For this analysis, the upstream regions of the 1,642 TSSs located in the 5'-UTR of annotated protein-coding genes were used. Conserved -10 motifs were detected in 1,619 sequences (98.6\%), whereas 1,616 of the analyzed sequences contributed to identification of the -35 motif (98.4\%). The conservation of a specific nucleotide at certain position is measured in bits and represented in the illustration by the size of the nucleotide. The hexamer of the core -10 region is underlined. The position values below the nucleotides are represented in relation to the positions of the identified TSSs, while the two spacers represent the mean distance between extended -10 region and TSS or -10 and -35 region, respectively. The depicted sequence logo was created with the software WebLogo [24].

\section{Determination of operon structures based on the whole transcriptome data set}

Using the paired-end sequencing reads from the whole transcriptome approach, an automated search for operon structures in B. methanolicus MGA3 was performed. The neighboring genes organized on the chromosome in sense direction were considered to compose a primary operon if they were connected by at least 4 combined read pairs. If TSSs were found within primary operon structures, suboperons were indicated. Upon manual analysis of the RNAseq data and by literature search, in 28 cases single genes were added to automatically detected operons. In total, 940 monocistronic transcripts were found in the transcriptome of B. methanolicus MGA3 and 1,164 genes were components of 381 operons with 94 suboperons (Figure 5). Thus, transcripts were detected for 2,092 of 3,330 (62.8\%) annotated genes in the genome of B. methanolicus MGA3. Most operons (60.5\%) consist of two genes and 12 operons comprised 8 or more genes (Table 2). The two largest operons each comprise 31 genes. The $f l g B$ operon is composed of 31 genes responsible for cell movement and chemotaxis in identical gene arrangement as found in B. subtilis [29]. The rpsJ operon with 26 genes coding for ribosomal proteins and five genes of various functions is also present in identical transcriptional gene organization in B. subtilis [30]. Only one of the 12 large operons, possibly involved in biosynthesis of amino sugars and their subsequent polymerization, does not have an equivalent in the genome of $B$. subtilis, but these genes can be found in Bacillus firmus, Anoxybacillus tepidamans and some Geobacillus species.

\section{Identification of novel transcripts in B. methanolicus MGA3}

Altogether, 525 TSSs belonging to novel transcripts (section "Detection of putative transcription start sites from

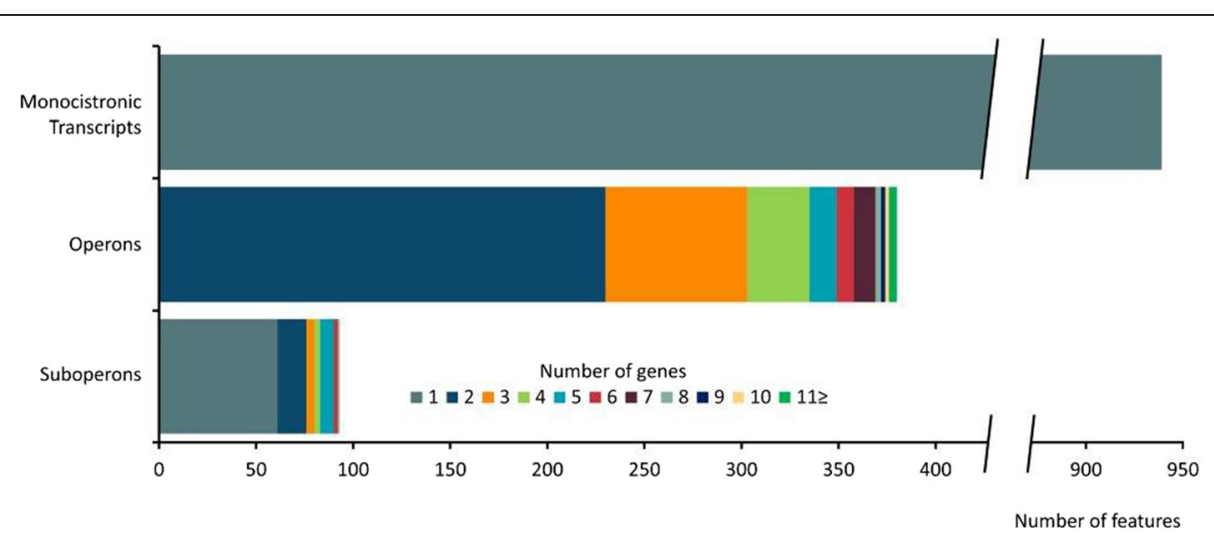

Figure 5 Analysis of operon structures and comparison of the number of genes assigned to monocistronic transcripts, primary operons and suboperons, identified in B. methanolicus MGA3. The bars represent the different categories of transcripts. Within each category the number of genes is highlighted with a color code as depicted in the legend below. 
Table 2 Largest identified primary operons on the B. methanolicus MGA3 chromosome

\begin{tabular}{|c|c|c|c|c|}
\hline Genes & $\begin{array}{l}\text { No. of } \\
\text { genes }\end{array}$ & Strand & Putative gene functions & $\begin{array}{l}\text { Transcriptional organization in } \\
\text { B. subtilis }{ }^{1}\end{array}$ \\
\hline $\begin{array}{l}\text { BMMGA3_00690- } \\
\text { BMMGA3_00840 }\end{array}$ & 31 & + & Various, mainly translation and ribosomal structure & Similar, [31] \\
\hline $\begin{array}{l}\text { BMMGA3_06100- } \\
\text { BMMGA3_06250 }\end{array}$ & 31 & + & Movement and chemotaxis & Similar, [29] \\
\hline $\begin{array}{l}\text { BMMGA3_01635- } \\
\text { BMMGA3_01690 }\end{array}$ & 12 & + & Purine biosynthesis & Similar, [32] \\
\hline $\begin{array}{l}\text { BMMGA3_15075- } \\
\text { BMMGA3_15130 }\end{array}$ & 12 & - & Amino sugars biosynthesis and polymerization & No similar operon present \\
\hline $\begin{array}{l}\text { BMMGA3_05780- } \\
\text { BMMGA3_05825 }\end{array}$ & 10 & + & Pyrimidine biosynthesis & Similar, [33] \\
\hline $\begin{array}{l}\text { BMMGA3_15975- } \\
\text { BMMGA3_16020 }\end{array}$ & 10 & - & ATP synthesis & {$[8 / 10]+1,[34]$} \\
\hline $\begin{array}{l}\text { BMMGA3_12730- } \\
\text { BMMGA3_12770 }\end{array}$ & 9 & - & Biosynthesis of branched amino acids & {$[8 / 9]+1,[35]$} \\
\hline $\begin{array}{l}\text { BMMGA3_01305- } \\
\text { BMMGA3_01345 }\end{array}$ & 9 & + & Control of SigB activity, general stress response & {$[7 / 9]+1,[36]$} \\
\hline $\begin{array}{l}\text { BMMGA3_02555- } \\
\text { BMMGA3_02595 }\end{array}$ & 9 & + & Sulfate reduction and activation, siroheme biosynthesis & {$[8 / 9]^{*}+2,[37]$} \\
\hline $\begin{array}{l}\text { BMMGA3_00415- } \\
\text { BMMGA3_00450 }\end{array}$ & 8 & + & Folic acid biosynthesis & {$[7 / 9]^{\S}[38]$} \\
\hline $\begin{array}{l}\text { BMMGA3_05870- } \\
\text { BMMGA3_05905 }\end{array}$ & 8 & + & $\begin{array}{l}\text { Various, mainly translation, ribosomal structure, signal } \\
\text { transduction }\end{array}$ & {$[3+3 / 7]^{\ddagger}+2,[39,40]$} \\
\hline $\begin{array}{l}\text { BMMGA3_14725- } \\
\text { BMMGA3_14760 }\end{array}$ & 8 & - & Histidine biosynthesis & Similar, [41] \\
\hline
\end{tabular}

${ }^{1}$ Genes in the same transcriptional organization as $B$. methanolicus MGA3 are depicted in square brackets. The number of transcripts that are present in the $B$. subtilis genome, but are not associated to the respective operon are indicated after the plus sign.

* In comparison to the $B$. methanolicus MGA3 the gene order is altered.

$\S$ In $B$. subtilis, the operon contains one additional gene.

${ }^{\ddagger}$ In $B$. subtilis, the genes are organized in two separate operons with.

RNA-seq data of enriched $5^{\prime}$ ends of primary transcripts") were detected by analysis of the primary transcriptome data set and classified into three categories according to their genomic context: intergenic (83), intragenic (227) or antisense (215). In addition, the whole transcriptome RNA-seq data were used to validate the novel transcripts and to determine their respective 3 'ends. When sequences downstream of the 83 intergenic TSSs were analyzed using ORF finder, BLAST and the Rfam database [42], 27 novel intergenic genes were identified, 24 of which coded for small RNAs (sRNA) of known or unknown function. Three novel genes encoding small and so far not annotated proteins in the B. methanolicus MGA3 genome were also found (Table 3 and Additional file 4: Table S4). Furthermore, 11 gene remnants with similarities to at least one homologous protein in another species were found (data not shown). Among the newly identified small RNAs was the small cytoplasmic RNA scRNA (named $4.5 \mathrm{~S}$ in E. coli). The scRNA identified in $B$. methanolicus MGA3 is approximately 337 nucleotides in length and is a member of the evolutionarily conserved signal-recognition-particle-like

Table 3 Novel transcripts with known function identified in B. methanolicus MGA3

\begin{tabular}{|c|c|c|c|c|c|c|}
\hline Name & Locus tag & Class & Feature start & Feature stop & Length & Strand \\
\hline 4.5S RNA/scRNA & BMMGA3_s0190 & SRNA & 27,291 & 27,628 & 307 & + \\
\hline RNase P RNA & BMMGA3_s0200 & sRNA & $2,087,761$ & $2,087,336$ & 425 & - \\
\hline 6S RNA/SsrS & BMMGA3_s0210 & sRNA & $2,278,029$ & $2,277,818$ & 211 & - \\
\hline $6 \mathrm{~S}$ RNA/SsrS & BMMGA3_s0220 & sRNA & $2,437,070$ & $2,436,840$ & 230 & - \\
\hline tmRNA/SsrA & BMMGA3_s0230 & sRNA & $2,890,631$ & $2,890,214$ & 417 & - \\
\hline SR4 RNA & BMMGA3_s0240 & sRNA & $1,925,025$ & $1,924,851^{*}$ & 174 & - \\
\hline$b s r G$ & BMMGA3_9642 & Protein & $1,924,777$ & $1,924,908$ & 132 & + \\
\hline
\end{tabular}

*The stop of this feature was approximated based on the published sequence in B. subtilis [44] and the terminator predication with ARNold [45]. 
RNA family [43]. Moreover, RNase P RNA (425 nt), two copies of the 6S RNA, tmRNA, which is part of the bacterial ribonucleoprotein complex, and the SR4 RNA, which belongs to a novel toxin-antitoxin system [44], were identified.

For 225 of the 227 intragenic TSSs an intragenic sense transcript was found (Additional file 5: Table S5). Interestingly, about one third of the intragenic TSSs were found within operons. Their promoter motifs were found to be nearly identical to the -35 and -10 elements recognized by the housekeeping sigma factor (data no shown).

For 152 of the 215 antisense TSSs transcripts longer than $20 \mathrm{nt}$ were found (Additional file 6: Table S6). Of those, 114 (75.0\%) were cis-antisense RNAs that overlap with coding regions on the complementary strand, 19 $(12.5 \%)$ were located antisense to a $5^{\prime}$ - or $3^{\prime}$-UTR of an annotated gene and 19 (12.5\%) were located antisense to a coding region and additionally overlapped the respective $5^{\prime}$ - or $3^{\prime}$-UTR. The 152 antisense genes are preceded by a conserved housekeeping sigma factor -10 motif, but lack conserved - 35 motifs (data no shown). The majority of them are genes of unknown function or only poorly characterized $(47 \%)$ or involved in metabolism (24\%). Interestingly, besides these 152 antisense genes, the genome of B. methanolicus contains 11 transcribed $5^{\prime}$-UTRs located antisense to the $5^{\prime}$-UTR or the coding region of a neighboring gene.

\section{Uncovering of cis-regulatory RNA elements by the analysis of $5^{\prime}$-UTRs}

Manual inspection of 342 identified 5'-UTRs longer than 100 nucleotides (the longest leader sequence was chosen if multiple TSSs were found) and comparison to the Rfam database [42] revealed 75 putative cis-regulatory elements present in 68 different 5 '-UTRs (Tables 4 , 5, 6 and 7). Remarkably, two consecutive cis-regulatory elements were present in seven $5^{\prime}$-UTRs. 41 putative riboswitches were found (Table 4) including those likely recognizing adenosylcobalamin (AdoCbl), flavin mononucleotide (FMN), S-adenosylmethionine (SAM), thiamine pyrophosphate (TPP), glycine, lysine, purines, 7-aminoethyl 7-deazaguanine (preQ1), cyclic diguanylate monophosphate (cyclic di-GMP), cyclic diadenylate monophosphate (c-di-AMP), and glucosamine-6phosphate (GlcN6P). Three putative cobalamin riboswitches were upstream of operons for cobalamin transport and biosynthesis, ribonucleotide-diphosphate reductase $\mathrm{NrdAB}$ and $\mathrm{a} \mathrm{B}_{12}$-independent methionine synthase (Table 4). Putative FMN riboswitches are located in front of the ribD operon for riboflavin biosynthesis and the $f m n P$ gene encoding a riboflavin transporter. The tenI and thiD operons, and thiW thiamine transporter gene are preceded by putative TPP riboswitches. Moreover, putative TPP riboswitches are located upstream of two similar versions of an operon containing taurine $\mathrm{ABC}$ transporter genes tau $B$ and tauC. Putative glycine riboswitches are located upstream of the glycine utilization operon $g c v T$ and the gene BMMGA3_03000 encoding an uncharacterized protein. A lysine riboswitch precedes the putative arginine/ornithine antiporter gene (Table 4). In B. methanolicus MGA3, 4 purine riboswitches were found upstream of the pur operon for de novo biosynthesis of purines, the $x p t-p b u X$ operon for xanthine salvage and catabolism, guaA encoding for GMP synthetase and the transporter gene $p b u G$. Putative PreQ1 riboswitches were identified upstream of the queC-queD-queE operon and the queF gene. Four putative c-di-GMP riboswitches precede genes of unknown function, the $\sin I$ gene encoding the antagonist of biofilm regulator $\operatorname{SinR}$ and the BMMGA3_04760-BMMGA3_04765 operon which is composed of genes participating in c-di-GMP turn-over. The presence of a $g \operatorname{lm} S$ riboswitch was detected in the 5'-UTR of $g l m S$ transcript.

Another major group of cis-regulatory elements in B. methanolicus MGA3 are T-boxes [46] that amount for $26.6 \%$ of all detected cis-regulatory RNA motifs (Table 5). Eighteen T-boxes were identified upstream of genes or operons encoding aminoacyl-tRNA synthetases, proteins involved in amino acid biosynthesis and transport (Table 5). Five auto-regulatory leader structures of ribosomal protein genes [47] were found (Table 6). Eleven further cis-regulatory RNA motifs were uncovered including a PyrR binding site preceding the pyr operon responsible for pyrimidine biosynthesis, pan, $y j d F$, and $y y b P-y k o Y$ RNA motifs upstream of the panB operon, $y j d F$ and $y k o Y$, respectively, as well as the $y l b H$ leader (Table 7).

\section{Transcript abundances of $B$. methanolicus MGA3}

All 3,225 loci for annotated chromosomal genes present in the genome were analyzed with respect to their transcript abundances in the cells. Normalization by calculating $\log$-RPKM values allowed comparison between genes as well as within one or different datasets (see section "Calculation of transcript abundances in Bacillus methanolicus MGA3"). Transcripts were detected for 2,529 of 3,225 gene loci (78.4\%). Genes that do not have an annotated function or encode hypothetical proteins are overrepresented among the non-transcribed genes (64.2\% of the non-transcribed as compared to $23.4 \%$ of all genes). Similarly, short ORFs up to 300 nt are overrepresented among the non-transcribed genes $(34.7 \%$ as compared to 11.1\%). Abundances of the transcribed genes were arbitrarily divided into 4 classes: 'Low', 'Middle,'High' and 'Very high'. The genes with 'Very high' transcript abundance constitute $1.3 \%$ of all chromosomal 
Table 4 Riboswitches identified in B. methanolicus MGA3 and their respective transcriptional organization, ligand and function

\begin{tabular}{|c|c|c|c|}
\hline Riboswitch and its transcriptional organization & Ligand & Related function & Locus tag(s) \\
\hline $\begin{array}{l}\text { (AdoCbl) -yvrC-yvrB-BMMGA3_01180-cobD- } \\
\text { BMMGA3_01190-cobU-cobQ }\end{array}$ & Adenosylcobalamin & $\begin{array}{l}\text { Cobalamin biosynthesis and } \\
\text { transport }\end{array}$ & $\begin{array}{l}\text { BMMGA3_01170- } \\
\text { BMMGA3_01200 }\end{array}$ \\
\hline (AdoCbl)-metE & & $\begin{array}{l}\mathrm{B}_{12} \text {-independent methionine } \\
\text { synthase }\end{array}$ & BMMGA3_08635 \\
\hline (AdoCbl)-nrdAB & & $\begin{array}{l}\text { Ribonucleotide-diphosphate } \\
\text { reductase }\end{array}$ & BMMGA3_09290 \\
\hline (FMN)-ribD-ribE-ribBA-ribH & Flavin mononucleotide & Riboflavin biosynthesis & $\begin{array}{l}\text { BMMGA3_09890- } \\
\text { BMMGA3_09905 }\end{array}$ \\
\hline$(\mathrm{FMN})-f m n P$ & & Riboflavin transport & BMMGA3_10940 \\
\hline (SAM)-BMMGA3_009040 & S-adenosylmethionine & Unknown function & BMMGA3_09040 \\
\hline (SAM)-metK & & S-adenosylmethionine synthesis & BMMGA3_13565 \\
\hline $\begin{array}{l}\text { (SAM)-cysH-sat-cysC-nirA-BMMGA3_02575-cobA-sirB1- } \\
\text { ytnM }\end{array}$ & & Sulfur assimilation & $\begin{array}{l}\text { BMMGA3_02555- } \\
\text { BMMGA3_02595 }\end{array}$ \\
\hline (SAM)-metN-metP-metQ & & Methionine $A B C$ transporter & $\begin{array}{l}\text { BMMGA3_14365- } \\
\text { BMMGA3_14375 }\end{array}$ \\
\hline (SAM)-mtnW-mtnX-mtnB-mtnD & & Methionine salvage & $\begin{array}{l}\text { BMMGA3_05045- } \\
\text { BMMGA3_05060 }\end{array}$ \\
\hline $\begin{array}{l}\text { (SAM)-BMMGA3_01840-BMMGA3_01845- } \\
\text { BMMGA3_01850 }\end{array}$ & & Unknown function & $\begin{array}{l}\text { BMMGA3_01840- } \\
\text { BMMGA3_01850 }\end{array}$ \\
\hline$(S A M)-d m o A$ & & DMS degradation & BMMGA3_09055 \\
\hline (SAM)-mtnK & & Methionine salvage & BMMGA3_05030 \\
\hline (SAM)-BMMGA3_08645-BMMGA3_08640 & & Unknown function & $\begin{array}{l}\text { BMMGA3_08640- } \\
\text { BMMGA3_08645 }\end{array}$ \\
\hline (SAM)-BMMGA3_03025 & & Methionine biosynthesis & BMMGA3_03025 \\
\hline (SAM)-BMMGA3_14380 & & Methionine biosynthesis & BMMGA3_14380 \\
\hline (SAM)-metl-metC-yitJ-metH & & Methionine biosynthesis & $\begin{array}{l}\text { BMMGA3_08600- } \\
\text { BMMGA3_08615 }\end{array}$ \\
\hline (SAM)-(PyrR)- BMMGA3_09265-BMMGA3_09270 & & $\begin{array}{l}\text { Major Facilitator Superfamily (MFS) } \\
\text { transporter }\end{array}$ & $\begin{array}{l}\text { BMMGA3_09265- } \\
\text { BMMGA3_09270 }\end{array}$ \\
\hline $\begin{array}{l}\text { (SAM)-(PyrR)-BMMGA3_03030-ssuA1-ssuC1-ssuB1-acsA1- } \\
\text { thrA }\end{array}$ & & Sulfonate uptake & $\begin{array}{l}\text { BMMGA3_03030- } \\
\text { BMMGA3_03055 }\end{array}$ \\
\hline (SAM)-(PyrR)-metN1-metP1-metQ1 & & Methionine $A B C$ transporter & $\begin{array}{l}\text { BMMGA3_08730- } \\
\text { BMMGA3_08740 }\end{array}$ \\
\hline (TPP)-thiD-thiE & Thiamine pyrophosphate & Biosynthesis of thiamine & $\begin{array}{l}\text { BMMGA3_03005- } \\
\text { BMMGA3_03010 }\end{array}$ \\
\hline (TPP)-tenA-tauB-tauC & & ABC transporter & $\begin{array}{l}\text { BMMGA3_07350- } \\
\text { BMMGA3_07360 }\end{array}$ \\
\hline (TPP)-thiW & & Thiamine uptake & BMMGA3_01780 \\
\hline (TPP)-tauA-BMMGA3_09465-tauC-tauB-tatD & & ABC transporter & $\begin{array}{l}\text { BMMGA3_09450- } \\
\text { BMMGA3_09470 }\end{array}$ \\
\hline (TPP)-tenl-thiO-thiS-thiG-moeB & & Biosynthesis of thiamine & $\begin{array}{l}\text { BMMGA3_01095- } \\
\text { BMMGA3_01115 }\end{array}$ \\
\hline (Gly)-gcvT-gcvPA-gcvPB & Glycine & Glycine utilization & $\begin{array}{l}\text { BMMGA3_11545- } \\
\text { BMMGA3_11555 }\end{array}$ \\
\hline (Gly)-BMMGA3_03000 & & Unknown function & BMMGA3_03000 \\
\hline (Lys)-BMMGA3_01150 & Lysine & Arginine/ornithine antiporter & BMMGA3_01150 \\
\hline (Pur)-pbuG & Purines & Hypoxanthine and guanine uptake & BMMGA3_01600 \\
\hline (Pur)-guaA & & Purine salvage & BMMGA3_01595 \\
\hline $\begin{array}{l}\text { (Pur)-purE-purK-purB-purC-purS-purQ-purL-purF-purM- } \\
\text { purN-purH }\end{array}$ & & Purine biosynthesis & $\begin{array}{l}\text { BMMGA3_01635- } \\
\text { BMMGA3_01690 }\end{array}$ \\
\hline
\end{tabular}


Table 4 Riboswitches identified in B. methanolicus MGA3 and their respective transcriptional organization, ligand and function (Continued)

\begin{tabular}{|c|c|c|c|}
\hline (Pur)-xpt-pbuX & & Xanthine salvage and catabolism & $\begin{array}{l}\text { BMMGA3_10440- } \\
\text { BMMGA3_10445 }\end{array}$ \\
\hline$\left(\right.$ PreQ $\left._{1}\right)$-queF & $\begin{array}{l}\text { 7-amminomethyl-7- } \\
\text { deazaguanine }\end{array}$ & Queuosine biosynthesis & BMMGA3_03100 \\
\hline$\left(\operatorname{PreQ}_{1}\right)$-queC-queD-queE & & Queuosine biosynthesis & $\begin{array}{l}\text { BMMGA3_09070- } \\
\text { BMMGA3_09080 }\end{array}$ \\
\hline (c-di-GMP)-BMMGA3_15625 & Cyclic di-GMP & Unknown function & BMMGA3_15625 \\
\hline (c-di-GMP)-BMMGA3_04760-BMMGA3_04765 & & Intracellular signal transduction & $\begin{array}{l}\text { BMMGA3_04760- } \\
B M M G A 3 \_04765\end{array}$ \\
\hline (c-di-GMP)-sinl & & Control of biofilm formation & BMMGA3_08510 \\
\hline (c-di-GMP)-BMMGA3_15380 & & Unknown function & BMMGA3_15380 \\
\hline (ydaO/yuaA)-BMMGA3_02260 & Cyclic di-AMP & Membrane protein & BMMGA3_02260 \\
\hline (ydaO/yuaA)-BMMGA3_14870 & & Amino acid permease & BMMGA3_14870 \\
\hline$(g \operatorname{lm} S)-g l m S$ & Glucosamine-6-phosphate & $\begin{array}{l}\text { Glutamine-fructose-6-phosphate } \\
\text { transaminase }\end{array}$ & BMMGA3_01020 \\
\hline
\end{tabular}

genes (Table 8). Of those 41 genes, 17 genes encode proteins related to transcription, translation or chaperone-assisted degradation, which is typical in bacteria [48]. Four genes with 'Very high' transcript abundance encode the methylotrophic enzymes 3hexulose-6-phosphate synthase (hps), 6-phospho-3- hexuloisomerase (phi), 6-phosphofructokinase (pfk) and ribose 5-phosphate isomerase (rpi). All those genes encode proteins of the RuMP and the high abundance of their transcripts may be due to the fact that methanol was used in most cultivations from which RNA was initially prepared for RNA-seq analysis.

Table 5 T-boxes identified in B. methanolicus MGA3 and their respective transcriptional organization, affected amino acid biosynthesis and related function

\begin{tabular}{|c|c|c|c|}
\hline $\begin{array}{l}\text { T-box and its transcriptional } \\
\text { organization }\end{array}$ & $\begin{array}{l}\text { Affected amino acid } \\
\text { biosynthesis }\end{array}$ & Related function & Locus tag(s) \\
\hline$(T-b o x)^{*}$-alas & Alanine & aaRS & BMMGA3_12250 \\
\hline gltX-(T-box)-cysE-cysS-mrnC-yacO-yacP & Cysteine & $\begin{array}{l}\text { Amino acid biosynthesis, aaRS, maturation of } \\
23 \mathrm{~S} \text { rRNA }\end{array}$ & $\begin{array}{l}\text { BMMGA3_00580- } \\
\text { BMMGA3_00605 }\end{array}$ \\
\hline (T-box)-glyQS & Glycine & aaRS & BMMGA3_11920 \\
\hline (T-box)-hisS-aspS & Histidine and aspartate & aaRS & $\begin{array}{l}\text { BMMGA3_12305- } \\
\text { BMMGA3_12310 }\end{array}$ \\
\hline (T-box)-iles & Isoleucine & aaRS & BMMGA3_05760 \\
\hline (T-box)-leus & Leucine & aaRS & BMMGA3_13510 \\
\hline (T-box)-phes & Phenylalanine & aaRS & BMMGA3_12925 \\
\hline (PyrR)(T-box)-prol & Proline & Amino acid biosynthesis & BMMGA3_11220 \\
\hline (PyrR)(T-box)-serS & Serine & aaRS & BMMGA3_00090 \\
\hline (T-box)-thrs & Threonine & aaRS & BMMGA3_12975 \\
\hline $\begin{array}{l}\text { (T-box)(T-box)-trpE-trpGD-trpD-trpF- } \\
\operatorname{trpB-trpA}\end{array}$ & Tryptophan & Amino acid biosynthesis & $\begin{array}{l}\text { BMMGA3_09595- } \\
\text { BMMGA3_09625 }\end{array}$ \\
\hline (T-box)-trpS & Tryptophan & aaRS & BMMGA3_04510 \\
\hline (T-box)-tyrs & Tyrosine & aaRS & BMMGA3_13290 \\
\hline (T-box)-vals & Valine & aaRS & BMMGA3_12655 \\
\hline (T-box)-brnQ & Branched amino acids & Amino acid transport & BMMGA3_02675 \\
\hline (T-box)-ilvB & Branched amino acids & Amino acid biosynthesis & BMMGA3_12765 \\
\hline (T-box)-cstAl & - & Carbon starvation protein ${ }^{\S}$ & BMMGA3_02765 \\
\hline
\end{tabular}

*(T-box $)=$ T-box regulatory element; $($ PyrR $)=$ PyrR binding site; aaRS = aminoacyl-tRNA synthetases; features of an operon are connected with a hyphen.

${ }^{\S}$ The whole transcriptome data does not unambiguously clarify if the T-box belongs to the cstA1 gene. 
Table 6 Ribosomal protein leaders identified in B. methanolicus MGA3 and their respective transcriptional organization and function

\begin{tabular}{|c|c|c|}
\hline $\begin{array}{l}\text { Ribosomal protein leaders and its transcriptional } \\
\text { organization }\end{array}$ & Related function & Locus tag(s) \\
\hline$(\mathrm{L} 10)^{*}-r p / J-r p / L$ & Ribosomal proteins & $\begin{array}{l}\text { BMMGA3_00640- } \\
\text { BMMGA3_00645 }\end{array}$ \\
\hline (L13)-rp/M-rps/ & Ribosomal proteins & $\begin{array}{l}\text { BMMGA3_00865- } \\
\text { BMMGA3_00870 }\end{array}$ \\
\hline (L19)-rp/S & Ribosomal protein & BMMGA3_06025 \\
\hline (L20)-infC-rpml-rp/T & $\begin{array}{l}\text { Translation initiation factor IF-3, ribosomal } \\
\text { proteins }\end{array}$ & $\begin{array}{l}\text { BMMGA3_12960- } \\
\text { BMMGA3_12970 }\end{array}$ \\
\hline (L21)-rp/U-BMMGA3_12500-rpmA & Ribosomal proteins, protein of unknown function & $\begin{array}{l}\text { BMMGA3_12495- } \\
\text { BMMGA3_12505 }\end{array}$ \\
\hline
\end{tabular}

$*(\mathrm{~L} 10)=\mathrm{L} 10$ leader; $(\mathrm{L} 13)=\mathrm{L} 13$ leader; $(\mathrm{L} 19)=\mathrm{L} 19$ leader; $(\mathrm{L} 20)=\mathrm{L} 20$ leader; $(\mathrm{L} 21)=\mathrm{L} 21$ leader.

\section{Discussion}

In the present study, the complete transcriptome of B. methanolicus MGA3 was analyzed for the first time with RNA sequencing. Previously, its genome including the plasmids pBM19 and pBM69 was sequenced $[11,16]$ and approximately 1000 proteins were characterized by label-free quantitative proteomics [8]. Here, a comprehensive characterization of transcription start sites, conserved sequence motifs of promoter and translation initiation sequences, the transcriptional organization of genes and putative regulatory RNA elements of B. methanolicus MGA3 using two different RNA-seq approaches is presented.

\section{Analysis of $5^{\prime}$-UTRs in $B$. methanolicus MGA3}

The distances of 1,642 TSSs to the next TLSs were equal or longer than 10 nucleotides in $>99 \%$ cases. The distance distribution showed a maximum at 26-35 nucleotides (22\%) before decreasing exponentially with increasing $5^{\prime}$-UTR lengths. This distance distribution is typical for bacteria that feature 5 '-UTRs shorter than $100 \mathrm{bp}$ and peak at a length of around 25-35 nt $[17,49,50]$. Similarly, the low number of leaderless transcripts in $B$. methanolicus MGA3 (6 with a $5^{\prime}$-UTR $\leq 10$ ) is in accordance with in silico predictions for 953 bacterial genomes that revealed only about $25 \%$ of species belonging to the phylum Firmicutes possess leaderless transcripts and in these species $2.4 \%$ to $16.6 \%$ of all sequences are leaderless [51]. Experimental evidence supports this notion (e.g. 2.1\% leaderless transcripts in Bacillus licheniformis [52]). Contrary, leaderless transcripts are abundant in species of the phyla Actinobacteria and Deinococcus-Thermus, e.g. Actinoplanes sp. SE50/110 (20\%) [49]. The exact translation mechanism of leaderless transcripts without a complete RBSs is still not fully elucidated, however, in those transcripts close proximity of the start codon to the 5 terminus is important for both ribosome binding and translation [53].

Characteristics of translation start sites, ribosome binding sites, promoters and operons in $B$. methanolicus MGA3

The most frequent initiation codon in $B$. methanolicus MGA3 is ATG (75.5\%), a frequency typical for 620 complete bacterial genomes (80.1\%; [54]). As in B. subtilis [55], the generally uncommon initiation codon TTG (13.9\%) was more common than GTG (10.6\%) in B. methanolicus MGA3.

Besides the initiation codon, the efficiency of translation depends on the interaction between the RBS and the 3 '-end of the $16 \mathrm{~S}$ rRNA [56]. The conserved RBS motif aGGaGg in B. methanolicus MGA3 is reverse complementary to the 3 '-end of the $16 \mathrm{~S}$ rRNA. The determined sequence motif is identical with the bacterial consensus sequence AGGAGG [57] and was found in

Table 7 Other cis-regulatory RNA motifs identified in B. methanolicus MGA3 and their respective transcriptional organization and function

\begin{tabular}{|c|c|c|}
\hline cis-regulatory RNA motif and its transcriptional organization & Related function & Locus tag(s) \\
\hline$(P y r R)^{*}$-pyrR-pyrP-pyrB-pyrC-pyrAA-pyrAB-pyrK-pyrD-pyrF-pyrE & Pyrimidine biosynthesis & BMMGA3_05780-BMMGA3_05825 \\
\hline (pan)-panB-panC-panD & Biosynthesis of coenzyme A & BMMGA3_10610- BMMGA3_10620 \\
\hline (yjdF)-yjdF & Unknown function & BMMGA3_03360 \\
\hline$(y y b P-y k o Y)(P y r R)-y k o Y$ & Membrane protein & BMMGA3_14085 \\
\hline$(y \mathrm{lbH})^{\S}$ & Unknown function & - \\
\hline
\end{tabular}

*(PyrR) = PyrR binding site; (pan) = pan RNA motif; (yjdF) = yjdF RNA motif; yybP-ykoY = yybP-ykoY RNA motif; (ylbH) =ylbH RNA motif.

${ }^{\S} \mathrm{A} C D S$ for a conserved hypothetical protein is located on the opposite strand between the ylbH RNA motif and the next downstream gene. 
Table 8 Highly abundant transcripts of B. methanolicus MGA3 ${ }^{a}$

\begin{tabular}{|c|c|c|c|}
\hline Gene name $^{b}$ & Gene product & log-RPKM & Functional category \\
\hline hps & 3-hexulose-6-phosphate synthase & 12,308 & Carbohydrate transport and metabolism \\
\hline phi & 3-hexulose-6-phosphate isomerase & 8,086 & Carbohydrate transport and metabolism \\
\hline rpiB & Ribose 5-phosphate isomerase & 3,473 & Carbohydrate transport and metabolism \\
\hline$p f k C$ & 6-phosphofructokinase & 1,695 & Carbohydrate transport and metabolism \\
\hline BMMGA3_16000 & FOF1 ATP synthase subunit B & 1,840 & Energy production and conversion \\
\hline yumB & $\mathrm{NADH}$ dehydrogenase-like protein YumB & 1,689 & Energy production and conversion \\
\hline$g / t B$ & Glutamate synthase [NADPH] small chain & 3,302 & Amino acid transport and metabolism \\
\hline$i l v E$ & Putative aminotransferase & 3,134 & Amino acid transport and metabolism \\
\hline $\arg G$ & Argininosuccinate synthase & 2,738 & Amino acid transport and metabolism \\
\hline $\arg F$ & Ornithine carbamoyltransferase & 2,434 & Amino acid transport and metabolism \\
\hline$i l v C$ & Ketol-acid reductoisomerase & 2,265 & Amino acid transport and metabolism \\
\hline $\arg B$ & Acetylglutamate kinase & 2,207 & Amino acid transport and metabolism \\
\hline $\operatorname{argH}$ & Argininosuccinate lyase & 2,187 & Amino acid transport and metabolism \\
\hline BMMGA3_12455 & L-aspartate oxidase & 2,028 & Amino acid transport and metabolism \\
\hline gltA & Glutamate synthase [NADPH] large chain & 1,780 & Amino acid transport and metabolism \\
\hline cysk & Cysteine synthase & 1,758 & Amino acid transport and metabolism \\
\hline BMMGA3_01295 & Putative transcriptional regulator, $\operatorname{CopG}$ family & 2,378 & Transcription \\
\hline rpoA & DNA-directed RNA polymerase subunit alpha & 1,670 & Transcription \\
\hline rpmC & $50 S$ ribosomal protein $\mathrm{L} 29$ & 7,670 & Translation \\
\hline BMMGA3_00355 & hypothetical protein & 4,650 & Translation \\
\hline$r p / R$ & $50 S$ ribosomal protein $\mathrm{L} 18$ & 4,412 & Translation \\
\hline$r p s G$ & 30 S ribosomal protein S7 & 3,468 & Translation \\
\hline rpl14e & $50 S$ ribosomal protein $\mathrm{L} 14 \mathrm{e}$ & 2,607 & Translation \\
\hline rps」 & $30 S$ ribosomal protein $\mathrm{S} 10$ & 2,511 & Translation \\
\hline$r p / s$ & $50 S$ ribosomal protein $\mathrm{L} 19$ & 2,249 & Translation \\
\hline rpss & 30 S ribosomal protein S19 & 2,204 & Translation \\
\hline$r p / W$ & $50 S$ ribosomal protein $\mathrm{L} 23$ & 2,004 & Translation \\
\hline$y b x F$ & $50 S$ ribosomal protein $\mathrm{L} 7 \mathrm{Ae}$ & 1,977 & Translation \\
\hline rpsU & 30 S ribosomal protein $\$ 21$ & 1,786 & Translation \\
\hline BMMGA3_12500 & hypothetical protein & 1,733 & Translation \\
\hline$r p / V$ & $50 S$ ribosomal protein $\mathrm{L} 22$ & 1,710 & Translation \\
\hline ykuU & Thioredoxin-like protein & 2,670 & Posttranslational modification, secretion, and vesicular transport \\
\hline groES & co-chaperonin GroES & 2,498 & Posttranslational modification, secretion, and vesicular transport \\
\hline sodA & Superoxide dismutase $[\mathrm{Mn}]$ & 2,511 & Inorganic ion transport and metabolism \\
\hline cysC & Adenylyl-sulfate kinase & 1,925 & Defense mechanisms \\
\hline BMMGA3_06005 & hypothetical protein & 5,873 & Function unknown \\
\hline ssb & Single-stranded DNA-binding protein & 4,261 & Function unknown \\
\hline BMMGA3_02575 & hypothetical protein (duf3906) & 1,757 & Function unknown \\
\hline BMMGA3_03940 & small, acid-soluble spore proteins & 1,728 & Function unknown \\
\hline my & Ribonuclease $Y$ & 2,180 & General function prediction only \\
\hline BMMGA3_15110 & acetyltransferase & 2,110 & General function prediction only \\
\hline
\end{tabular}

${ }^{a}$ Transcript abundance was arbitrarily classified into 5 classes. The RNA abundance is reflected by the log-RPKM value. The data set revealed $21.6 \%$ nontranscribed genes, 3.8\% genes with low RNA abundance (log-RPKM >0-16), 41.2\% genes with middle (log-RPKM >16-160), 32.2\% genes with high (log-RPKM $>160-1600$ ) and $1.3 \%$ genes with very high (log-RPKM $>1600$ ) transcript abundance.

${ }^{\mathrm{b}}$ The genes are sorted according to their functional category. 
$21.9 \%$ of the upstream regions. The first three bases of the motif are conserved in $66.9 \%$ of the upstream sequences, which is in line with the notion that the first three bases of the RBS are potentially more relevant to the translation initiation via hybridization to the 3 '-terminus of the $16 \mathrm{~S}$ rRNA, while the other bases possibly only modulate the translation efficiency [58]. The spacing between translation starts and ribosome binding sites is known to affect translation efficiency [58]. In B. methanolicus MGA3, the distance between both elements was between 5 to 10 nucleotides (97.1\%) with an average of 7.4 bases, thus, matching the optimal spacing determined for E. coli, B. subtilis and various other bacteria $[59,60]$.

Conserved promoter motifs were determined upstream of the exact TSSs positions within the B. methanolicus MGA3 and revealed a -10 region (TAtaaT), which is identical to the -10 consensus sequence TATAAT originally described for E. coli $\sigma^{70}$-RNAP [61] and is also recognized by the $B$. subtilis $\sigma^{\mathrm{A}}$-RNAP [62]. The finding that TGN is preceding $33 \%$ of the -10 motifs in B. methanolicus MGA3 is in accordance with extended -10 motifs of $\sigma^{70} / \sigma^{\mathrm{A}}$-dependent promoters in E. coli and B. subtilis with the dinucleotide TG located 1 bp upstream of the core hexamer $[63,64]$. Extended -10 promoters specifically interact with the conserved sigma factor region 3.0 to form the polymerasepromoter complex and often lack the -35 consensus element [65]. While it is assumed that the additional contact with the sigma factor in extended -10 promoters compensates for the interaction between the region 4.2 and the -35 element [66], the -35 sequence in $B$. methanolicus was not particularly less conserved if a TGN extended -10 region was present. The so-called discriminator element downstream of the -10 hexamer may play a role in the regulation of the stringent response during transition from exponential to stationary phase [67] and nucleotides at positions -6 to -4 interact with the sigma factor region 1.2 [68]. However, in B. methanolicus MGA3 only two consecutive adenines are weakly conserved, which reflects the overall low $G+C$ content of this bacterium $(38.6 \%)$. The mean spacing of the identified -10 regions to the TSS is with 6.7 bases in typical range for bacteria $[30,63]$. The average spacing of $16.6 \mathrm{nt}$ between the -10 and the -35 regions is very close to 17 , the optimal spacing determined for these elements in $E$. coli and B. subtilis [63,69]. The weakly conserved -35 hexamer ttgana determined for B. methanolicus MGA3 resembles the -35 consensus sequence TTGACA present in E. coli, B. subtilis and numerous other species $[69,70]$.

Although various different stress conditions were included in the experimental setup, the analysis of conserved promoter motifs in B. methanolicus MGA3 only returned sequences that are identical to the consensus sequences recognized by bacterial housekeeping sigma factors. This is likely due to the fact that in all tested conditions the number of housekeeping genes accounts for most of the identified TSSs and thus overlays the more rare upstream regions of stress-induced transcripts. Since pooled RNAs were sequenced, it was not possible to differentiate between transcripts which were resulting from a certain cultivation condition. To identify sigma factor-dependent promoter motifs, RNA-seq data sets for individual growth or stress conditions need to be generated and analyzed; however, this was not within the scope of the present study.

In B. methanolicus MGA3, 381 primary operons (encompassing 1,164 genes) with 94 internal suboperons were identified, while 940 genes were transcribed monocistronically under the chosen growth/stress conditions. Although this distribution is typical and also found in E. coli, B. subtilis and C. glutamicum $[17,71,72]$, it is important to mention here that the transcriptional organization of genes is very likely not a static feature necessitating the analysis of RNA-seq data sets for individual growth or stress conditions.

\section{Description of novel transcripts in B. methanolicus MGA3}

The novel transcripts detected in $B$. methanolicus MGA3 were classified as intergenic, intragenic or antisense transcripts (Table 3 and Additional file 4: Table S4; Additional file 5: Table S5 and Additional file 6: Table S6). The 27 novel intergenic transcripts represent small RNA and small protein genes, but their function still has to be elucidated. For six small RNAs (sRNA) and one small protein of B. methanolicus MGA3 a function could be predicted via database searches. The scRNA (337 nucleotides in B. methanolicus MGA3) is a member of the signal-recognition-particle-like RNA family [43]. Together with the Ffh protein component (encoded in B. methanolicus MGA3 by BMMGA3_05995), the scRNA constitutes the bacterial signal recognition particle (SRP), which targets proteins for secretion by directing them to the translocation channels in the membrane [73]. The RNase P is an essential and ubiquitous ribozyme, mainly responsible the maturation of tRNA molecules [74]. Bacterial RNase P consists of two components: RNase P RNA, which is the catalytic subunit, and the C5 protein which assists in the release of the product from the holoenzyme [75] and is encoded in B. methanolicus MGA3 by BMMGA3_16755. The 6S RNA, which typically regulates transcription by forming a stable complex with the housekeeping form of the RNAP holoenzyme [76], is present in two copies in the B. methanolicus MGA3 genome, which is consistent with findings from $B$. subtilis and several closely related bacteria [77]. Intriguingly, these two copies are 
differentially expressed in these organisms, which suggests their involvement at different stages of growth [77]. B. methanolicus MGA3 possesses transfermessenger RNA (tmRNA) as one of the most abundant RNA species, similarly to other bacterial species [78]. The tmRNA can act as a tRNA and mRNA and constitutes a ribonucleoprotein complex together with the three protein components SmpB, EF-Tu and (usually) the ribosomal protein S1, while the latter is absent from bacilli and is substituted by a homologue encoded by the $y p f D$ gene [79]. The bacterial ribonucleoprotein complex resets stalled ribosomes and a tmRNA-encoded signal peptide is cotranslationally appended to the C-termini of nascent polypeptide and serves as a recognition signal for degradation [80]. The tmRNA open reading frame in B. methanolicus MGA3 encodes a 16 amino acid (aa) signal peptide with a terminal stop codon (KTSKPITGNQKLALAA-Stop), which resembles the 15 aa sequence identified in $B$. subtilis (AGKTNSFNQNVALAA-Stop) [81]. B. methanolicus MGA3 may possess a type I toxin-antitoxin system with toxin-encoding gene $b s r G$ and the SR4 RNA as initially described in B. subtilis [44]. In B. subtilis, the small RNA SR4 promotes the degradation of the bsrG mRNA via the double-strand specific RNase III, while the absence of SR4 lead to the overexpression of $b s r G$ and subsequently to cell lysis and growth retardation [44]. A comparable function may be predicted for B. methanolicus MGA3 since bsrG and SR4 RNA are located antisense to each other with an overlap of ca. 120 bases and the 43 aa toxin encoded by $b s r G$ in $B$. methanolicus MGA3 (MTAVLQHRRSLAIVV PAGVRPMKQDRPLPQFAVKGGLFILVKS-Stop) shares $19 \%$ of identity and $31 \%$ of similarity with the 38 aa toxin (MTVYESLMIMINFGGLILNTVLLIFNIMMIVTS SQKKK-Stop) present in B. subtilis.

The frequent occurrence of intragenic TSSs (10\%) in B. methanolicus MGA3 that almost exclusively (99\%) belong to intragenic sense transcripts preceded by conserved -35 and -10 motifs for the housekeeping sigma factor is not unusual in bacteria. Notably, about one third of the internal sense transcripts in B. methanolicus MGA3 belong to suboperons, thus, the remaining downstream coding sequences are transcribed completely. The functional role of the remaining intragenic transcripts, for example as alternative, shorter proteins, novel or processed RNAs which increase the transcriptomic complexity, is not clear and should be further analyzed.

Altogether, 152 antisense transcripts longer than $20 \mathrm{nt}$ were identified in 146 different protein-coding genes of B. methanolicus MGA3. Extrapolated, $4.4 \%$ of the B. methanolicus MGA3 genes or more may be subject to antisense transcription. This number is in accordance with findings for other species from the genus Bacillus
[52] and other transcriptome studies which suggest that antisense transcription probably affects $5-20 \%$ of bacterial genes and in some cases even considerably more, i.e. about $46 \%$ in Helicobacter pylori [82]. A conserved housekeeping -10 motif was identified for the antisense transcripts in B. methanolicus MGA3 in their upstream regions. By contrast, neither a conserved -35 motif nor an extended -10 motifs were found for these antisense transcripts. Antisense transcripts may regulate gene expression either by hybridization with part or the complete target sequence and e.g. blocking/releasing ribonuclease cleavage or ribosome binding sites [83] or competing with transcription of sense and antisense sequences [84]. It is predicted that transcription of 11 antisense genes of $B$. methanolicus MGA3 interferes with transcription of the neighboring gene due to overlapping 5 '-UTRs. Antisense transcription has been detected in all domains of life and therefore likely represents a common form of gene expression regulation [83,85]. Although, RNA-seq studies often reveal large numbers of antisense transcripts, further validation and functional characterization is necessary to identify if, and to what extent, those antisense RNAs participate in gene regulation.

\section{Discovery of cis-regulatory RNA elements in the B. methanolicus MGA3 genome}

Cis-regulatory RNA elements belong to broad class of non-coding RNA motifs which are present upstream of the regulated genes. In the present study, 75 cis-regulatory elements were detected in 68 different $5^{\prime}$-UTRs in the transcriptome of B. methanolicus MGA3. The RNAseq data of $B$. methanolicus reveal three cobalamin riboswitches upstream of genes/operons predicted to encode cobalamin biosynthesis- and transport-related proteins, $B_{12}$-independent methionine synthase and ribonucleotide-diphosphate reductase. Likely, they function as demonstrated for the cobalamin riboswitches upstream of the nrdABS operon of Streptomyces coelicolor and of the $\mathrm{B}_{12}$-independent methionine synthase of Mycobacterium tuberculosis [86,87]. The organization of FMN, SAM, TPP, lysine and purine riboswitches found in the genome of B. methanolicus MGA3 is similar to those of other bacteria [88-91]. The function of the riboswitch belonging to the $g c v T$ operon in the genome of B. methanolicus has been confirmed experimentally for $B$. subtilis. A next ligand for the riboswitch found in the genome of $B$. methanolicus is $\mathrm{PreQ}_{1}$ (7-aminomethyl-7-deazaguanosine) which is a precursor of nucleoside Q (queuosine). This modified guanine nucleotide present in some tRNA molecules is known to participate in the regulation of genes involved in queuosine biosynthesis by interaction with $\mathrm{PreQ}_{1}$ riboswitches in other bacterial species $[92,93]$. The intracellular signal 
molecule bis- $\left(3^{\prime}-5^{\prime}\right)$-cyclic dimeric guanosine monophosphate (c-di-GMP) is found in many bacteria and may be involved e.g. in regulation of motility, virulence and biofilm formation (as reviewed in [94]) and c-di-GMP riboswitches have been characterized [95]. Besides presence of putative c-di-GMP riboswitches the genome of B. methanolicus encodes at least 8 enzymes for synthesis and degradation of c-di-GMP (BMMGA3_05195, BMMGA3_05210, BMMGA3_09025, BMMGA3_10495, BMMGA3_12645, BMMGA3_13275, BMMGA3_14250 and BMMGA3_15230). The ydaO/yuaA leader was shown to interact with cyclic di-AMP in B. subtilis [96]. This motif is present upstream of two genes of B. methanolicus MGA3, BMMGA3_02260 and BMMG $A 3$ 14870, which encode a membrane protein and an amino acid permease, respectively. Unlike the other riboswitches mentioned above, the glmS ribozyme, which is present in B. methanolicus MGA3, is both a ribozyme, catalyzing a chemical reaction, and a riboswitch, regulating transcription of the glutaminefructose-6-phosphate transaminase gene as a function of the glucosamine-6-phosphate (GlcN6P) concentration [97]. This type of regulation involves self-cleavage of the $\operatorname{glm} S$ ribozyme in the presence of GlcN6P. Although homologs are found in at least 17 further Gram-positive bacteria the function of the predicted $g \operatorname{lm} S$ riboswitch in B. methanolicus has yet to be proven [97].

In addition to the riboswitches, one of the most abundant regulatory elements discovered for $B$. methanolicus are T-box motifs which likely control gene expression by interacting with uncharged tRNA molecules. The binding of uncharged tRNA prevents formation of a translation terminator and, thus enables translation [98]. T-boxes were found in several monocistronic and polycistronic transcripts which feature genes encoding aminoacyl-tRNA synthetases (aaRS) or proteins involved in amino acid biosynthesis and transport in B. methanolicus MGA3. A tandem T-box was detected for the trpE operon which is also characteristic for certain Bacillales and some related species such as Clostridium beijerinckii and Desulfitobacterium hafniense [98]. The operon hisSasp $S$ represents an unusual case of two aaRS genes controlled by only one riboswitch. It has been suggested that this regulatory element can adopt two alternative secondary structures and therefore sense both tRNA $\mathrm{H}_{\text {His }}$ and $\mathrm{tRNA}_{\text {Asp }}$ molecules [99]. Interestingly, the T-box was also found upstream of the gene coding for carbon starvation protein CstA1. However, the detailed investigation of whole transcriptome data for upstream region of the cstA1 gene indicates that the T-box in fact might not regulate the CstA1 expression and is only a residue of a different transcription unit which was split in the process of genome rearrangements. This hypothesis is based on the fact that there is a break in the transcription between the TSS and the gene start codon in the whole transcriptome track. However, this assumption has yet to be confirmed experimentally.

Besides low-molecular-weight compounds, also proteins such as the ribosomal proteins L10, L13, L20, L21 and L29 are able to bind to the leader sequences of their own transcripts causing premature transcription termination at the leader terminator [100-102]. In B. methanolicus MGA3, five leader motifs characteristic for the genes of above-mentioned ribosomal proteins were detected. Additionally, a PyrR binding site was found in the leader sequence of the pyr operon. PyrR is encoded by and auto-regulates the pyrimidine biosynthesis operon by formation of a terminator and decrease of operon expression in B. subtilis [33].

In seven cases, B. methanolicus features two cis-regulatory RNA elements in the same upstream region: a duplicated T-box and in six cases the PyrR binding site was accompanied by either a SAM riboswitch (three times), a T-box (twice) or the $y y b P-y k o Y$ leader (once). The presence of two consecutive cis-regulatory elements was observed in different bacterial species where it was proposed that the tandem arrangement leads to an increase of the regulatory capacity $[103,104]$. Interestingly, no known regulatory RNA element was found in the second longest leader sequence detected in the transcriptome of B. methanolicus MGA3. This 5'-UTR of 878 nt length belongs to the gene encoding molybdopterin dehydrogenase FAD-binding protein. A nucleotide BLAST analysis of the leader sequence showed only partial similarity to the upstream region of molybdopterin dehydrogenase FAD-binding protein gene in the Geobacillus stearothermophilus NUB3621 genome. However, because of the low conservation of the motif among bacterial species no conclusions about the function of the long leader can be drawn and further experiments need to be performed to understand its role in the transcriptome of B. methanolicus MGA3.

\section{The mRNA abundances in B. methanolicus MGA3 reflect its metabolic organization}

Under the chosen conditions, $21.6 \%$ of the chromosomal genes of $B$. methanolicus MGA3 were not transcribed. The RNA-seq data were generated from a pool of RNA preparations from various conditions, however, the different lab conditions most likely reflect only a small subset of the conditions encountered by B. methanolicus in its natural habitat. Alternatively, since genes for hypothetical proteins are about three times more abundant in the group of non-transcribed genes $(64.2 \%)$ than in the group of genes for which transcripts were detected (23.4\%), false annotations of protein-coding genes may have increased the fraction of non-transcribed genes. 
As a first approximation to categorize expression levels, all chromosomal genes of B. methanolicus MGA3 were divided into four groups according to arbitrarily chosen transcripts abundance thresholds (section "Transcript abundances of B. methanolicus MGA3"). The 41 genes showing the highest RNA abundance encoded proteins of (1) amino acid metabolism and transport, (2) transcription, translation and post-translational modification, (3) carbohydrates metabolism and energy production, and (4) other functions. This representation of the gene functions is expected and to some extend corresponds to the results obtained on proteome level for B. subtilis [105]. Notably, genes relevant for methylotrophy of B. methanolicus MGA3 stood out. Although, the results obtained in the present study do not allow to perform the direct comparison of transcripts abundance between different references (chromosome, plasmids pBM19 and pBM69) [106], the pBM19-encoded $m d h$ has the most abundant transcript among all pBM19encoded genes, while the chromosome-encoded methanol dehydrogenase genes have rather low transcript abundances. This finding is in accordance with qRTPCR experiments and the proteome analyses that showed that the pBM19-encoded $m d h$ transcript and gene product is more abundant than those of the chromosomal genes $m d h 2$ (60 -fold) and $m d h 3$ (4000fold) [106]. Formaldehyde, a toxic intermediate generated by methanol oxidation, is assimilated via 3-hexulose-6phosphate synthase and 6-phospho-3-hexuloisomerase. As expected, the co-transcribed hps and phi genes showed the highest RNA abundance of all the chromosomal genes. For 6-phospofructokinase which catalyzes the next step in the RuMP cycle, high transcript abundance for the chromosomal gene was observed. Transcripts of the chromosomal genes encoding fructose 1,6-bisphosphate aldolase [14], transketolase [12], fructose 1,6-bisphosphatase [13] and ribose 5-phosphate epimerase belong to the transcript group with high abundance. The last reaction of the RuMP cycle is catalyzed by ribose-5-phosphate isomerase, which is encoded only on the chromosome. As for $h p s$ and phi that are also only encoded on the chromosome, rpi RNA was very abundant. Interestingly, the gltAB operon encoding glutamate synthase was also found among the highly abundant genes ( $g l t A$ and gltB); $B$. methanolicus MGA3 has two alternative active glutamate synthases [106] and this finding may suggest that the gltAB encoded enzyme is important for the high production of L-glutamate by this bacterium. In future work aimed to gain an in-depth understanding of methylotrophy in B. methanolicus, it will be imperative to combine transcriptome, proteome and metabolome experiments under carefully controlled conditions of growth with methanol versus a non-methylotrophic carbon source.

\section{Conclusion}

In this study, RNA-seq data sets have been generated for the primary and the whole transcriptome of the thermophilic methylotrophic Bacillus methanolicus MGA3. Altogether, 1,642 TSSs in the upstream regions of annotated genes were identified and used for analysis of the 5 '-UTR length distribution and the detection of 75 cis-regulatory RNA elements. Additionally, the exact TSSs were used to detect conserved sequence motifs for TLSs, RBSs and promoters. Examination of the whole transcriptome enabled the validation of 365 novel transcripts, the uncovering of 381 operons and the determination of the mRNA abundance in B. methanolicus MGA3. The data established in this study deliver valuable insight regarding various transcriptomic elements and represent the basis for further transcriptome studies of B. methanolicus MGA3. Moreover, detailed insight into promoter and translation initiation sequences should be valuable for the future development of new and better expression tools for B. methanolicus.

\section{Availability of supporting data}

The data sets supporting the results of this article are available in the NCBI Gene Expression Omnibus database, under the accession number GSE64469, http:// www.ncbi.nlm.nih.gov/geo/query/acc.cgi?acc=GSE64469.

\section{Additional files}

Additional file 1: Table S1. Primers used for RNA quality control.

Additional file 2: Table S2. Bacillus methanolicus MGA3 cultivation parameters.

Additional file 3: Table S3. List of Bacillus methanolicus MGA3 CDS with corrected translational start sites.

Additional file 4: Table S4. Novel transcripts identified in Bacillus methanolicus MGA3 with unknown function.

Additional file 5: Table S5. Identified intragenic sense transcripts of Bacillus methanolicus MGA3.

Additional file 6: Table S6. Identified antisense transcripts of Bacillus methanolicus MGA3.

\section{Competing interests}

The authors declare that they have no competing interests.

\section{Authors' contributions}

$\mathrm{MI}$ and AN carried out the experimental procedure, the complete data analysis of the present study and also prepared a draft of the manuscript. CR performed the mapping of raw RNA-seq reads to the reference sequences. $T B, J K$ and VFW coordinated the study and helped finalize the manuscript. All authors read and approved the manuscript.

\section{Acknowledgements}

The authors acknowledge support of the publication fee by Deutsche Forschungsgemeinschaft and the Open Access Publication Funds of Bielefeld University. The authors thank Timo Wolf for assistance with the bioreactor cultivations and our colleagues Anika Winkler and Andreas Albersmeier for sequencing of the cDNA libraries. Additionally, we thank Rolf Hilker and Prof. Alexander Goesmann (Bioinformatics and Systems Biology, Gießen University, Germany) for providing and constantly improving the RNA-seq visualization 
and evaluation tool ReadXplorer [22]. In this context, the authors also thank Juri Ritter (Bioinformatics Resource Facility, CeBiTec) for developing a ReadXplorer module for the bioinformatical analysis of RNA-seq data. $\mathrm{Ml}$ and AN acknowledge support from the CLIB Graduate Cluster Industrial Biotechnology at Bielefeld University, Germany which is supported by a grant from the Federal Ministry of Innovation, Science and Research (MIWF) of the federal state North Rhine-Westphalia, Germany. $\mathrm{MI}, \mathrm{TB}$ and VFW acknowledge support of the FP7 EU project PROMYSE (289540).

\section{Author details}

${ }^{1}$ Genetics of Prokaryotes, Faculty of Biology \& Center for Biotechnology, Bielefeld University, Universitätsstr. 25, 33615 Bielefeld, Germany. ${ }^{2}$ Microbial Genomics and Biotechnology, Center for Biotechnology, Bielefeld University, Universitätstr. 27, 33615 Bielefeld, Germany. ${ }^{3}$ Department of Molecular Biology, SINTEF Materials and Chemistry, Sem Selands vei 2, 7465 Trondheim, Norway. ${ }^{4}$ Department of Biotechnology, Norwegian University of Science and Technology, Sem Sælands vei 6/8, 7491 Trondheim, Norway. ${ }^{5}$ Technology Platform Genomics, Center for Biotechnology, Bielefeld University, Universitätsstr. 27, 33615 Bielefeld, Germany.

Received: 17 September 2014 Accepted: 12 January 2015 Published online: 14 February 2015

\section{References}

1. Arfman N, Dijkhuizen L, Kirchhof G, Ludwig W, Schleifer K, Bulygina ES, et al. Bacillus methanolicus sp. nov., a new species of thermotolerant, methanol-utilizing, endospore-forming bacteria. Int J Syst Bacteriol. 1992;42:439-45

2. Schendel F, Bremmon C, Flickinger M, Guettler M, Hanson R. L-Lysine production at $50^{\circ} \mathrm{C}$ by mutants of a newly isolated and characterized methylotrophic Bacillus sp. Appl Environ Microbiol. 1990;56:963-70.

3. Schendel F, Dillingham R, Hanson RS, Sano K, Matsui K. Production of glutamate using wild type Bacillus methanolicus. Patent (US6083728). 2000:1-20

4. Brautaset T, Williams MD, Dillingham RD, Kaufmann C, Bennaars A, Crabbe E, et al. Role of the Bacillus methanolicus citrate synthase II gene, citY, in regulating the secretion of glutamate in L-Lysine-secreting mutants. Appl Environ Microbiol. 2003;69:3986-95.

5. Schrader J, Schilling M, Holtmann D, Sell D, Filho MV, Marx A, et al. Methanol-based industrial biotechnology: current status and future perspectives of methylotrophic bacteria. Trends Biotechnol. 2009;27:107-15.

6. Brautaset $T$, Jakobsen $\varnothing \mathrm{M}$, Josefsen $\mathrm{KD}$, Flickinger MC, Ellingsen TE. Bacillus methanolicus: a candidate for industrial production of amino acids from methanol at $50^{\circ} \mathrm{C}$. Appl Microbiol Biotechnol. 2007;74:22-34.

7. Arfman N, Watling E, Clement W, van Oosterwijk R, de Vries G, Harder W, et al. Methanol metabolism in thermotolerant methylotrophic Bacillus strains involving a novel catabolic NAD-dependent methanol dehydrogenase as a key enzyme. Arch Microbiol. 1989;152:280-8.

8. Müller JEN, Litsanov B, Bortfeld-Miller M, Trachsel C, Grossmann J, Brautaset $T$, et al. Proteomic analysis of the thermophilic methylotroph Bacillus methanolicus MGA3. Proteomics. 2014;14:725-37.

9. Ochsner AM, Müller JEN, Mora CA, Vorholt JA. In vitro activation of NADdependent alcohol dehydrogenases by Nudix hydrolases is more widespread than assumed. FEBS Lett. 2014;588:2993-9.

10. Brautaset $T$, Jakobsen $\varnothing \mathrm{M}$, Flickinger MC, Valla S, Ellingsen TE. PlasmidDependent methylotrophy in thermotolerant Bacillus methanolicus. J Bacteriol. 2004;186:1229-38.

11. Heggeset TMB, Krog A, Balzer S, Wentzel A, Ellingsen TE, Brautaset T. Genome sequence of thermotolerant Bacillus methanolicus: features and regulation related to methylotrophy and production of L-lysine and L-glutamate from methanol. Appl Environ Microbiol. 2012;78:5170-81.

12. Markert B, Stolzenberger J, Brautaset T, Wendisch VF. Characterization of two transketolases encoded on the chromosome and the plasmid pBM19 of the facultative ribulose monophosphate cycle methylotroph Bacillus methanolicus. BMC Microbiol. 2014;14:7.

13. Stolzenberger J, Lindner SN, Persicke M, Brautaset T, Wendisch VF Characterization of fructose 1,6-bisphosphatase and sedoheptulose 1 , 7-bisphosphatase from the facultative ribulose monophosphate cycle methylotroph Bacillus methanolicus. J Bacteriol. 2013;195:5112-22.
14. Stolzenberger J, Lindner SN, Wendisch VF. The methylotrophic Bacillus methanolicus MGA3 possesses two distinct fructose 1,6-bisphosphate aldolases. Microbiology. 2013;159(Pt 8):1770-81.

15. Jakobsen ØM, Benichou A, Flickinger MC, Valla S, Ellingsen TE, Brautaset $T$. Upregulated transcription of plasmid and chromosomal ribulose monophosphate pathway genes is critical for methanol assimilation rate and methanol tolerance in the methylotrophic bacterium Bacillus methanolicus. J Bacteriol. 2006;188:3063-72.

16. Irla M, Neshat A, Winkler A, Albersmeier A, Heggeset TMB, Brautaset T, et al. Complete genome sequence of Bacillus methanolicus MGA3, a thermotolerant amino acid producing methylotroph. J Biotechnol. 2014; S0168-1656:00818-9.

17. Pfeifer-Sancar K, Mentz A, Rückert C, Kalinowski J. Comprehensive analysis of the Corynebacterium glutamicum transcriptome using an improved RNAseq technique. BMC Genomics. 2013;14:888.

18. Sharma C, Hoffmann S, Darfeuille F, Reignier J, Findeiss S, Sittka A, et al. The primary transcriptome of the major human pathogen Helicobacter pylori. Nature. 2010;464(March):250-5.

19. Jakobsen $\varnothing \mathrm{M}$, Brautaset $T$, Degnes KF, Heggeset TMB, Balzer S, Flickinger $M C$, et al. Overexpression of wild-type aspartokinase increases L-lysine production in the thermotolerant methylotrophic bacterium Bacillus methanolicus. Appl Environ Microbiol. 2009;75:652-61.

20. Kircher M, Stenzel U, Kelso J. Improved base calling for the Illumina Genome Analyzer using machine learning strategies. Genome Biol. 2009;10:R83.

21. Blom J, Jakobi T, Doppmeier D, Jaenicke S, Kalinowski J, Stoye J, et al. Exact and complete short-read alignment to microbial genomes using Graphics Processing Unit programming. Bioinformatics. 2011;27:1351-8.

22. Hilker R, Stadermann KB, Doppmeier D, Kalinowski J, Stoye J, Straube J, et al. Genome analysis ReadXplorer - Visualization and Analysis of Mapped Sequences. Bioinformatics. 2014;30:2247-54.

23. Ao W, Gaudet J, Kent WJ, Muttumu S, Mango SE. Environmentally induced foregut remodeling by PHA-4/FoxA and DAF-12/NHR. Science. 2004;305:1743-6.

24. Schneider T, Stephens R. Sequence logos: a new way to display consensus sequences. Nucleic Acids Res. 1990;18:6097-100.

25. Mortazavi A, Williams BA, McCue K, Schaeffer L, Wold B. Mapping and quantifying mammalian transcriptomes by RNA-Seq. Nat Methods. 2008:5:1-8.

26. Ai-Awadhi N, Egli T, Hamer G. Applied Microbiology Biotechnology Growth characteristics of a thermotolerant methylotrophic Bacillus sp. (NCIB 12522) in batch culture. Appl Microbiol Biotechnol. 1988;29:485-93.

27. Bastet L, Dubé A, Massé E, Lafontaine DA. New insights into riboswitch regulation mechanisms. Mol Microbiol. 2011;80:1148-54.

28. Lewis P, Partridge SR, Errington J. Sigma factors, asymmetry, and the determination of cell fate in Bacillus subtilis. Proc Natl Acad Sci U S A. 1994;91:3849-53.

29. Albertini AM, Caramori T, Crabb WD, Scoffone F, Galizzi A. The flaA locus of Bacillus subtilis is part of a large operon coding for flagellar structures, motility functions, and an ATPase-like polypeptide. J Bacteriol. 1991;173:3573-9.

30. Liu J, Turnbough CL. Effects of transcriptional start site sequence and position on nucleotide-sensitive selection of alternative start sites at the pyrC promoter in Escherichia coli. J Bacteriol. 1994;176:2938-45.

31. Li X, Lindahl L, Sha Y, Zengel JM. Analysis of the Bacillus subtilis S10 ribosomal protein gene cluster identifies two promoters that may be responsible for transcription of the entire 15-kilobase S10-spc-alpha cluster. J Bacteriol. 1997;179:7046-54.

32. Ebbole D, Zalkin H. Cloning and characterization of a 12-gene cluster from Bacillus subtilis encoding nine enzymes for de novo purine nucleotide synthesis. J Biol Chem. 1987;262:8274-87.

33. Turner RJ, Lu Y, Switzer RL. Regulation of the Bacillus subtilis pyrimidine biosynthetic (pyr) gene cluster by an autogenous transcriptional attenuation mechanism. J Bacteriol. 1994;176:3708-22.

34. Presecan E, Moszer I, Boursier L, Cruz RH, de la Fuente V, Hullo M, et al. The Bacillus subtilis genome from gerBC (311 degrees) to licR (334 degrees). Microbiology. 1997;143(Pt 10):3313-28.

35. Grandoni JA, Zahler SA, Calvo JM. Transcriptional regulation of the ilv-leu operon of Bacillus subtilis. J Bacteriol. 1992;174:3212-9.

36. Wise AA, Price CW. Four additional genes in the $\operatorname{sig} B$ operon of Bacillus subtilis that control activity of the general stress factor sigma $B$ in response to environmental signals. J Bacteriol. 1995;177:123-33. 
37. Mansilla MC, Albanesi D, de Mendoza D. Transcriptional control of the sulfur-regulated cysH operon, containing genes involved in L-cysteine biosynthesis in Bacillus subtilis. J Bacteriol. 2000;182:5885-92.

38. De Saizieu A, Vankan P, Vockler C, van Loon A. The trp RNA-binding attenuation protein (TRAP) regulates the steady-state levels of transcripts of the Bacillus subtilis folate operon. Microbiology. 1997;143(Pt 3):979-89.

39. Hinc K, Iwanicki A, Seror S, Obuchowski M. Mapping of a transcription promoter located inside the priA gene of the Bacillus subtilis chromosome. Acta Biochim Pol. 2006;53:497-505.

40. Iwanicki A, Hinc K, Seror S, Wegrzyn G, Obuchowski M. Transcription in the prpC-yloQ region in Bacillus subtilis. Arch Microbiol. 2005;183:421-30.

41. Mäder U, Homuth G, Scharf C, Büttner K, Bode R, Hecker M. Transcriptome and proteome analysis of Bacillus subtilis gene expression modulated by amino acid availability. J Bacteriol. 2002;184:4288-95.

42. Griffiths-Jones S, Bateman A, Marshall M, Khanna A, Eddy SR. Rfam: an RNA family database. Nucleic Acids Res. 2003;31:439-41.

43. Nakamura K, Hashizume E, Shibata T, Nakamura Y, Mala S, Yamane K. Small cytoplasmic RNA (scRNA) gene from Clostridium perfringens can replace the gene for the Bacillus subtilis scRNA in both growth and sporulation. Microbiology. 1995;141(Pt 11):2965-75.

44. Jahn N, Preis H, Wiedemann C, Brantl S. BsrG/SR4 from Bacillus subtilis-the first temperature-dependent type I toxin-antitoxin system. Mol Microbiol. 2012;83:579-98.

45. Naville M, Ghuillot-Gaudeffroy A, Marchais A, Gautheret D. ARNold: A web tool for the prediction of Rho-independent transcription terminators. RNA Biol. 2011;8:11-3.

46. Grundy FJ, Rollins SM, Henkin TM. Interaction between the acceptor end of tRNA and the T box stimulates antitermination in the Bacillus subtilis tyrs gene: a new role for the discriminator base. J Bacteriol. 1994;176:4518-26.

47. Nomura M, Yates J. Feedback regulation of ribosomal protein gene expression in Escherichia coli: structural homology of ribosomal RNA and ribosomal protein mRNA. Proc Natl Acad Sci U S A. 1980;77:7084-8.

48. Karlin S, Mrázek J. Predicted highly expressed genes of diverse prokaryotic genomes. J Bacteriol. 2000;182:5238-50.

49. Schwientek P, Neshat A, Kalinowski J, Klein A, Rückert C, Schneiker-Bekel S, et al. Improving the genome annotation of the acarbose producer Actinoplanes sp. SE50/110 by sequencing enriched $5^{\prime}$-ends of primary transcripts. J Biotechnol. 2014;190:85-95.

50. Schmidtke C, Findeiss S, Sharma CM, Kuhfuss J, Hoffmann S, Vogel J, et al. Genome-wide transcriptome analysis of the plant pathogen Xanthomonas identifies sRNAs with putative virulence functions. Nucleic Acids Res. 2012;40:2020-31.

51. Zheng X, Hu G-Q, She Z-S, Zhu H. Leaderless genes in bacteria: clue to the evolution of translation initiation mechanisms in prokaryotes. BMC Genomics. 2011;12:361.

52. Wiegand S, Dietrich S, Hertel R, Bongaerts J, Evers S, Volland S, et al. RNASeq of Bacillus licheniformis: active regulatory RNA features expressed within a productive fermentation. BMC Genomics. 2013;14:667.

53. Krishnan KM, Van Etten WJ, Janssen GR. Proximity of the start codon to a leaderless mRNA's 5 ' terminus is a strong positive determinant of ribosome binding and expression in Escherichia coli. J Bacteriol. 2010;192:6482-5.

54. Villegas A, Kropinski AM. An analysis of initiation codon utilization in the Domain Bacteria - concerns about the quality of bacterial genome annotation. Microbiology. 2008;154(Pt 9):2559-661.

55. Moszer I. The complete genome of Bacillus subtilis: from sequence annotation to data management and analysis. FEBS Lett. 1998:430:28-36

56. Ozbudak EM, Thattai M, Kurtser I, Grossman AD, van Oudenaarden A. Regulation of noise in the expression of a single gene. Nat Genet. 2002;31:69-73.

57. Shine J, Dalgarno L. The 3'-terminal sequence of Escherichia coli $16 \mathrm{~S}$ ribosomal RNA: complementarity to nonsense triplets and ribosome binding sites. Proc Natl Acad Sci U S A. 1974;71:1342-6.

58. Ringquist S, Shinedling S, Barrick D, Green L, Binkley J, Stormo G, et al. Translation initiation in Escherichia coli: sequences within the ribosome-binding site. Mol Microbiol. 1992;6:1219-29.

59. Vellanoweth $R$, Rabinowitz J. The influence of ribosome-binding-site elements on translational efficiency in Bacillus subtilis and Escherichia coli in vivo. Mol Microbiol. 1992;6:1105-14.

60. Li G-W, Oh E, Weissman JS. The anti-Shine-Dalgarno sequence drives translational pausing and codon choice in bacteria. Nature. 2012;484:538-41.
61. Hawley D, McClure W. Compilation and analysis of Escherichia coli promoter DNA sequences. Nucleic Acids Res. 1983;11:2237-55.

62. Camacho A, Salas M. Effect of mutations in the "extended -10 " motif of three Bacillus subtilis sigmaA-RNA polymerase-dependent promoters. J Mol Biol. 1999;286:683-93.

63. Helmann JD. Compilation and analysis of Bacillus subtilis sigma A-dependent promoter sequences: evidence for extended contact between RNA polymerase and upstream promoter DNA. Nucleic Acids Res. 1995;23:2351-60

64. Burr T, Mitchell J, Kolb A, Minchin S, Busby S. DNA sequence elements located immediately upstream of the-10 hexamer in Escherichia coli promoters: a systematic study. Nucleic Acids Res. 2000;28:1864-70.

65. Campbell EA, Muzzin O, Chlenov M, Sun JL, Olson CA, Weinman O, et al. Structure of the Bacterial RNA Polymerase Promoter Specificity $\sigma$ Subunit. Mol Cell. 2002;9:527-39.

66. Kumar A, Malloch R, Fujita N, Smillie D, Ishihama A, Hayward R. The minus 35-recognition region of Escherichia coli sigma 70 is inessential for initiation of transcription at an"extended minus 10" promoter. J Mol Biol. 1993:232:406-18.

67. Lamond Al, Travers AA. Genetically separable functional elements mediate the optimal expression and stringent regulation of a bacterial tRNA gene. Cell. 1985;40:319-26.

68. Haugen SP, Berkmen MB, Ross W, Gaal T, Ward C, Gourse RL. rRNA promoter regulation by nonoptimal binding of sigma region 1.2: an additional recognition element for RNA polymerase. Cell. 2006;125:1069-82.

69. Ishihama A. Promoter selectivity of prokaryotic RNA polymerases. Trends Genet. 1988:4:282-6.

70. Moran CP, Lang N, Banner CD, Haldenwang WG, Losick R. Promoter for a developmentally regulated gene in Bacillus subtilis. Cell. 1981;25:783-91.

71. Gama-Castro S, Jiménez-Jacinto V, Peralta-Gil M, Santos-Zavaleta A, Peñaloza-Spinola MI, Contreras-Moreira B, et al. RegulonDB (version 6.0): gene regulation model of Escherichia coli K-12 beyond transcription, active (experimental) annotated promoters and Textpresso navigation. Nucleic Acids Res. 2008;36(Database issue):D120-4.

72. Sierro N, Makita Y, de Hoon M, Nakai K. DBTBS: a database of transcriptional regulation in Bacillus subtilis containing upstream intergenic conservation information. Nucleic Acids Res. 2008;36(Database issue):D93-6.

73. Peluso P, Herschlag D, Nock S, Freymann DM, Johnson AE, Walter P. Role of 4.5S RNA in Assembly of the Bacterial Signal Recognition Particle with its Receptor. Science. 2000;288:1640-3.

74. Hartmann E, Hartmann RK. The enigma of ribonuclease P evolution. Trends Genet. 2003;19:561-9.

75. Sun L, Campbell FE, Zahler NH, Harris ME. Evidence that substrate-specific effects of $C 5$ protein lead to uniformity in binding and catalysis by RNase $P$. EMBO J. 2006;25:3998-4007.

76. Brownlee G. Sequence of 6S RNA of E. coli. Nature. 1971;229:147-9.

77. Barrick J, Sudarsan N, Weinberg Z, Ruzzo WL, Breaker RR. 6S RNA is a widespread regulator of eubacterial RNA polymerase that resembles an open promoter. RNA. 2005;11:774-84.

78. Andini N, Nash K. Expression of tmRNA in mycobacteria is increased by antimicrobial agents that target the ribosome. FEMS Microbiol Lett. 2011;322:172-9.

79. Isono K, Isono S. Lack of ribosomal protein S1 in Bacillus stearothermophilus. Proc Natl Acad Sci U S A. 1976:73:3-6.

80. Gottesman S, Roche E, Zhou Y, Sauer RT. The ClpXP and ClpAP proteases degrade proteins with carboxy-terminal peptide tails added by the SsrA-tagging system. Genes Dev. 1998;12:1338-47.

81. Ito K, Tadaki T, Lee S, Takada K, Muto A, Himeno H. Trans-translation mediated by Bacillus subtilis tmRNA. FEBS Lett. 2002;516:245-52.

82. Sharma CM, Vogel J. Differential RNA-seq: the approach behind and the biological insight gained. Curr Opin Microbiol. 2014;19C:97-105.

83. Thomason M, Storz G. Bacterial antisense RNAs: How many are there and what are they doing? Annu Rev Genet. 2010;44:167-88.

84. Pelechano V, Steinmetz LM. Gene regulation by antisense transcription. Nat Rev Genet. 2013;14:880-93.

85. Storz G, Vogel J, Wassarman K. Regulation by small RNAs in bacteria: expanding frontiers. Mol Cell. 2011;43:880-91.

86. Borovok I, Gorovitz B, Schreiber R, Aharonowitz Y, Cohen G. Coenzyme B12 controls transcription of the Streptomyces class la ribonucleotide reductase nrdABS operon via a riboswitch mechanism. J Bacteriol. 2006;188:2512-20. 
87. Warner DF, Savvi S, Mizrahi V, Dawes SS. A Riboswitch Regulates Expression of the Coenzyme B12-Independent Methionine Synthase in Mycobacterium tuberculosis: Implications for Differential Methionine Synthase Function in Strains H37Rv and CDC1551. J Bacteriol. 2007;189:3655-9.

88. McDaniel B, Grundy F, Kurlekar VP, Tomsic J, Henkin TM. Identification of a mutation in the Bacillus subtilis S-adenosylmethionine synthetase gene that results in derepression of S-box gene expression. J Bacteriol. 2006;188:3674-81.

89. Grundy FJ, Henkin TM. The S box regulon: a new global transcription termination control system for methionine and cysteine biosynthesis genes in gram-positive bacteria. Mol Microbiol. 1998;30:737-49.

90. Auger S, Yuen WH, Danchin A, Martin-Verstraete I. The metIC operon involved in methionine biosynthesis in Bacillus subtilis is controlled by transcription antitermination. Microbiology. 2002;148(Pt 2):507-18.

91. Hullo M-F, Auger S, Dassa E, Danchin A, Martin-Verstraete I. The metNPQ operon of Bacillus subtilis encodes an ABC permease transporting methionine sulfoxide, D- and L-methionine. Res Microbiol. 2004;155:80-6.

92. Okada N, Noguchi S, Nishimura S, Ohgi T, Goto T, Crain PF, et al. Structure determination of a nucleoside $\mathrm{Q}$ precursor isolated from E. coli tRNA: 7-\{aminomethyl)-7-deazaguanosine. Nucleic Acids Res. 1978:5:2289-96.

93. Roth A, Winkler WC, Regulski EE, Lee BWK, Lim J, Jona I, et al. A riboswitch selective for the queuosine precursor preQ1 contains an unusually small aptamer domain. Nat Struct Mol Biol. 2007;14:308-17.

94. Hengge R. Principles of c-di-GMP signalling in bacteria. Nat Rev Microbiol. 2009; 7:263-73.

95. Sudarsan N, Lee ER, Weinberg Z, Moy RH, Kim JN, Link KH, et al. Riboswitches in eubacteria sense the second messenger cyclic di-GMP. Science. 2008;321:411-3.

96. Nelson JW, Sudarsan N, Furukawa K, Weinberg Z, Wang JX, Breaker RR. Riboswitches in eubacteria sense the second messenger c-di-AMP. Nat Chem Biol. 2013;9:834-9.

97. Winkler WC, Nahvi A, Roth A, Collins JA, Breaker RR. Control of gene expression by a natural metabolite-responsive ribozyme. Nature. 2004:428:281-6.

98. Vitreschak AG, Mironov AA, Lyubetsky VA, Gelfand MS. Comparative genomic analysis of T-box regulatory systems in bacteria. RNA. 2008;14:717-35.

99. Gutiérrez-Preciado A, Henkin TM, Grundy FJ, Yanofsky C, Merino E. Biochemical features and functional implications of the RNA-based T-box regulatory mechanism. Microbiol Mol Biol Rev. 2009;73:36-61.

100. Choonee N, Even S, Zig L, Putzer H. Ribosomal protein L20 controls expression of the Bacillus subtilis infC operon via a transcription attenuation mechanism. Nucleic Acids Res. 2007:35:1578-88.

101. Johnsen M, Christensen T, Dennis PP, Fiil NP. Autogenous control: ribosomal protein L10-L12 complex binds to the leader sequence of its mRNA. EMBO J. 1982;1:999-1004.

102. Yao Z, Barrick J, Weinberg Z, Neph S, Breaker R, Tompa M, et al. A computational pipeline for high- throughput discovery of cis-regulatory noncoding RNA in prokaryotes. PLoS Comput Biol. 2007;3:e126.

103. Sudarsan N, Hammond MC, Block KF, Welz R, Barrick JE, Roth A, et al. Tandem riboswitch architectures exhibit complex gene control functions. Science. 2006;314:300-4.

104. Gutierrez-Preciado A, Jensen RA, Yanofsky C, Merino E. New insights into regulation of the tryptophan biosynthetic operon in Gram-positive bacteria. Trends Genet. 2005;21:432-6.

105. Bairoch A, Apweiler R. The SWISS-PROT protein sequence database and its supplement TrEMBL in 2000. Nucleic Acids Res. 2000;28:45-8.

106. Krog A, Heggeset TMB, Müller JEN, Kupper CE, Schneider O, Vorholt JA et al. Methylotrophic Bacillus methanolicus encodes two chromosomal and one plasmid born $\mathrm{NAD}^{+}$dependent methanol dehydrogenase paralogs with different catalytic and biochemical properties. PLoS One. 2013;8: e59188.

\section{Submit your next manuscript to BioMed Central and take full advantage of:}

- Convenient online submission

- Thorough peer review

- No space constraints or color figure charges

- Immediate publication on acceptance

- Inclusion in PubMed, CAS, Scopus and Google Scholar

- Research which is freely available for redistribution 